\title{
LLAMA: The $M_{\mathrm{BH}}-\sigma_{\star}$ relation of the most luminous local AGNs
}

\author{
Turgay Caglar ${ }^{1}$, Leonard Burtscher ${ }^{1}$, Bernhard Brandl ${ }^{1}$, Jarle Brinchmann ${ }^{1,2}$, Richard I. Davies ${ }^{3}$, Erin K. S. Hicks ${ }^{4}$,
} Michael Koss ${ }^{5}$, Ming-Yi Lin ${ }^{6}$, Witold Maciejewski ${ }^{7}$, Francisco Müller-Sánchez ${ }^{8}$, Rogemar A. Riffel ${ }^{9}$, Rogério Riffel $^{10}$, David J. Rosario ${ }^{11}$, Marc Schartmann ${ }^{3,12}$, Allan Schnorr-Müller ${ }^{10}$, T. Taro Shimizu ${ }^{3}$, Thaisa Storchi-Bergmann ${ }^{10,13}$, Sylvain Veilleux ${ }^{14,15}$, Gilles Orban de Xivry ${ }^{16}$, and Vardha N. Bennert ${ }^{17}$

${ }^{1}$ Leiden Observatory, PO Box 9513, 2300 RA Leiden, The Netherlands e-mail: caglar@strw.leidenuniv.nl

2 Instituto de Astrofísica e Ciências do Espaço, Universidade do Porto, CAUP, Rua das Estrelas, 4150-762 Porto, Portugal

3 Max-Planck-Institut für Extraterrestrische Physik, Postfach 1312, 85741 Garching, Germany

4 Department of Physics \& Astronomy, University of Alaska Anchorage, Anchorage, AK 99508-4664, USA

5 Eureka Scientific Inc, Oakland, CA, USA

${ }^{6}$ Institute of Astronomy and Astrophysics, Academia Sinica, 11F of AS/NTU Astronomy-Mathematics Building, No.1, Sec. 4, Roosevelt Rd, Taipei 10617, Taiwan

7 Astrophysics Research Institute, Liverpool John Moores University, IC2 Liverpool Science Park, 146 Brownlow Hill, Liverpool L3 5RF, UK

8 Center for Astrophysics and Space Astronomy, University of Colorado, Boulder, CO 80309-0389, USA

9 Universidade Federal de Santa Maria, Departamento de Física/CCNE, 97105-900 Santa Maria, RS, Brazil

10 Departamento de Astronomia, Universidade Federal do Rio Grande do Sul, IF, CP 15051, 91501-970 Porto Alegre, RS, Brazil

11 Centre for Extragalactic Astronomy, Department of Physics, Durham University, South Road, Durham DH1 3LE, UK

12 Universitäts-Sternwarte München, Scheinerstraße 1, 81679 München, Germany

13 Harvard-Smithsonian Center for Astrophysics, 60 Garden St., Cambridge, MA 02138, USA

14 Department of Astronomy and Joint Space-Science Institute, University of Maryland, College Park, Maryland 20742, USA

15 Institute of Astronomy and Kavli Institute for Cosmology Cambridge, University of Cambridge, Cambridge CB3 OHA, UK

16 Space Sciences, Technologies, and Astrophysics Research Institute, Université de Liège, 4000 Sart Tilman, Belgium

17 Department of Physics, California Polytechnic State University, San Luis Obispo, CA 93407, USA

Received 15 July 2019 / Accepted 13 December 2019

\section{ABSTRACT}

Context. The $M_{\mathrm{BH}}-\sigma_{\star}$ relation is considered a result of coevolution between the host galaxies and their supermassive black holes. For elliptical bulge hosting inactive galaxies, this relation is well established, but there is still discussion concerning whether active galaxies follow the same relation.

Aims. In this paper, we estimate black hole masses for a sample of 19 local luminous active galactic nuclei (AGNs; LLAMA) to test their location on the $M_{\mathrm{BH}}-\sigma_{\star}$ relation. In addition, we test how robustly we can determine the stellar velocity dispersion in the presence of an AGN continuum and AGN emission lines, and as a function of signal-to-noise ratio.

Methods. Supermassive black hole masses $\left(M_{\mathrm{BH}}\right)$ were derived from the broad-line-based relations for $\mathrm{H} \alpha, \mathrm{H} \beta$, and $\mathrm{Pa} \beta$ emission line profiles for Type 1 AGNs. We compared the bulge stellar velocity dispersion $\left(\sigma_{\star}\right)$ as determined from the Ca II triplet (CaT) with the dispersion measured from the near-infrared CO (2-0) absorption features for each AGN and find them to be consistent with each other. We applied an extinction correction to the observed broad-line fluxes and we corrected the stellar velocity dispersion by an average rotation contribution as determined from spatially resolved stellar kinematic maps.

Results. The $\mathrm{H} \alpha$-based black hole masses of our sample of AGNs were estimated in the range $6.34 \leq \log M_{\mathrm{BH}} \leq 7.75 M_{\odot}$ and the $\sigma_{\star \text { СаT }}$ estimates range between $73 \leq \sigma_{\star \mathrm{CaT}} \leq 227 \mathrm{~km} \mathrm{~s}^{-1}$. From the so-constructed $M_{\mathrm{BH}}-\sigma_{\star}$ relation for our Type 1 AGNs, we estimate the black hole masses for the Type 2 AGNs and the inactive galaxies in our sample.

Conclusions. We find that our sample of local luminous AGNs is consistent with the $M_{\mathrm{BH}}-\sigma_{\star}$ relation of lower luminosity AGNs and inactive galaxies, after correcting for dust extinction and the rotational contribution to the stellar velocity dispersion.

Key words. accretion, accretion disks - black hole physics - galaxies: active - galaxies: bulges - galaxies: evolution galaxies: Seyfert

\section{Introduction}

Theoretical and observational evidence in the last decade has shown that supermassive black holes (SMBHs) reside in the majority of galaxy nuclei and play a substantial role in the evolution of galaxies. Lynden-Bell (1969) recognized that SMBHs primarily grow via mass accretion, during which an extreme amount of energy is released. Nowadays, it is widely accepted that active galactic nuclei (AGNs) are powered by mass accretion onto SMBHs via the conversion of gravitational energy into radiation through accretion disks (e.g., Padovani et al. 2017, and references therein). The feeding of SMBHs begins with materials accretion at extragalactic scales, which subsequently passes through galactic and nuclear scales to the broad-line region (BLR) and accretion disk before falling into the black hole or being ejected by jets or winds 
(Storchi-Bergmann \& Schnorr-Müller 2019). The materials in the host galaxy residing near the nucleus can be ionized by radiation (e.g., Davidson 1972; Netzer et al. 1990). Spectral studies have confirmed the existence of two distinct regions of excited gas clouds near the nucleus, referred as the BLR and the narrowline region (NLR). The BLR gas resides at subparsec scales, whereas NLR gas can be found up to a few kiloparsec from the central black hole (Netzer 1990).

Detailed investigations of the BLR became possible in the last few decades as a result of large dedicated observing campaigns (e.g., Blandford \& McKee 1982; Peterson 1993; Onken \& Peterson 2002; Denney et al. 2006, 2010; Bentz et al. 2006, 2009a, 2016; Grier et al. 2012, 2013a). These campaigns have allowed the interaction between the SMBH and surrounding gas clouds to be characterized in detail. Under virial equilibrium, it is possible to use the BLR gas as an estimator for SMBH mass using the line widths of rotation-broadened emission lines. Even though virial black hole masses $\left(M_{\mathrm{BH}}\right)$ are roughly consistent with masses derived from other methods (e.g., Peterson et al. 2004; Peterson 2007), there are a few complications, namely the structure, kinematics, and orientation of the BLR. To obtain accurate black hole masses, it is fundamental to know these BLR properties. Application of the virial theorem allows us to use the emission line width of the BLR gas as a tracer of BLR rotational velocity. While the radius of the BLR is inferred from reverberation mapping (RM), other efforts to resolve the structure, kinematics, and orientation of the BLR have been limited so far (Pancoast et al. 2014; Grier et al. 2017); but new instrumentation developments have facilitated recent progress to resolve the BLR directly (GRAVITY Collaboration 2018). Correspondingly, these parameters have been used for estimating black hole masses of AGNs.

A growing body of evidence suggests a tight connection between the evolution and formation of SMBHs and host galaxies (e.g., Ferrarese \& Merritt 2000; Tremaine et al. 2002; Merritt \& Ferrarese 2001; Gebhardt et al. 2000; Ferrarese \& Ford 2005; Gültekin et al. 2009; Beifiori et al. 2012; McConnell \& Ma 2013; Kormendy \& Ho 2013). This tight connection suggests that host galaxy properties, such as stellar velocity dispersion and/or bulge mass, can be used a proxy for black hole mass. The observational present-day black hole mass-galaxy comparisons, i.e., black hole mass - stellar velocity dispersion $\left(M_{\mathrm{BH}}-\sigma_{\star}\right)$, show very strong correlations for inactive galaxies, which are hosting elliptical bulges (e.g., McConnell \& Ma 2013; Kormendy \& Ho 2013, hereafter MM13, KH13, respectively). This tight relation is usually attributed to evidence that feedback mechanisms must be responsible for linking the growth of galaxy bulges to accretion, although the exact feedback mechanism is still under debate. Using the observational data, the $M_{\mathrm{BH}}-\sigma_{\star}$ relation has been parameterized as a power-law function with index $\alpha\left(M_{\mathrm{BH}} \propto \sigma^{\alpha}\right)$, where $\alpha$ was found to be between 3 and 6 . From a theoretical concept, the difference between the power-law index is attributable to different feedback models: momentumdriven or energy-driven winds, which expects an $\alpha=4$ (King 2003) and $\alpha=5$ (Silk \& Rees 1998) relation, respectively. In these models, shocked shells of matter are driven outward by winds; correspondingly, the galaxy bulges grow via the central star formation. In both models, AGN accretion must approach the Eddington limit to form winds that can blow gas out of the host galaxy. In case of major mergers, a larger amount of gas can be driven onto the SMBH, and fueling of black holes can lead to a coupled SMBH-bulge growth. But, coevolution can occur relatively slowly in the case of secular evolution, which results in the formation of pseudo-bulges. Even though the
$M_{\mathrm{BH}}-\sigma_{\star}$ correlation is very tight for the galaxies hosting elliptical bulges, galaxies with pseudo-bulges are reported to lie below the $M_{\mathrm{BH}}-\sigma_{\star}$ relation (e.g., Greene et al. 2010; Kormendy et al. 2011; Kormendy \& Ho 2013).

The assumption that AGNs and inactive galaxies follow the same $M_{\mathrm{BH}}-\sigma_{\star}$ relation is still under debate. In previous studies, Nelson et al. (2004), Onken et al. (2004), and Yu \& Lu (2004) investigated the $M_{\mathrm{BH}}-\sigma_{\star}$ relation of AGNs; unfortunately, their measurements suffered from low-quality data and an unreliable $M_{\mathrm{BH}}-\sigma \star$ relation for inactive galaxies. Afterward, Greene \& Ho (2006a) found an intrinsic scatter of 0.61 dex from the $M_{\mathrm{BH}}-\sigma_{\star}$ relation for local AGNs using the RM and singleepoch black hole masses. Accordingly, Woo et al. (2010, 2013, 2015), Graham et al. (2011), Park et al. (2012a) and Batiste et al. (2017a) reported shallower $M_{\mathrm{BH}}-\sigma_{\star}$ relations for reverberationmapped AGNs. But the resulting discrepancy between active and inactive galaxies was assumed to be related to unreliable $\sigma_{\star}$ calculations of AGNs and/or the lack of AGNs in the high $\mathrm{SMBH}$ mass regime. Unfortunately, the number of high $\mathrm{SMBH}$ masses $\left(M_{\mathrm{BH}}>10^{8} M_{\odot}\right)$ from reverberation-mapped AGNs was too low to make a direct comparison with the inactive sample. To increase the number of the AGNs, other studies concentrated on single-epoch SMBH mass estimations, but a few large offsets $\left(>0.5\right.$ dex) from the inactive $M_{\mathrm{BH}}-\sigma \star$ relation were also reported from the single-epoch based investigations (Barth et al. 2005; Greene \& Ho 2006a; Shen et al. 2008; Subramanian et al. 2016; Koss et al. 2017). Thus, the intrinsic scatter from inactive $M_{\mathrm{BH}}-\sigma_{\star}$ relation remains highly uncertain for AGNs.

To calibrate the $M_{\mathrm{BH}}-\sigma_{\star}$ scaling relation, black hole masses are mostly determined by modeling stellar kinematics or spatially resolving gas for galaxies in the local universe. On the other hand, black hole masses are determined via RM or megamaser disks for AGNs. In RM-based estimations, a dimensionless scale factor $f$ is required to convert the virial product into black holes, and it is estimated assuming an average multiplicative offset from the $M_{\mathrm{BH}}-\sigma \star$ relation for AGN-hosting galaxies (Onken et al. 2004). Although the $M_{\mathrm{BH}}-\sigma_{\star}$ relation appears to be tight, the slope of the relation remains uncertain (i.e., the slope of both AGN and/or inactive samples). Previous studies reported significantly different slopes of the $M_{\mathrm{BH}}-\sigma_{\star}$ relation for AGNs with respect to the $M_{\mathrm{BH}}-\sigma_{\star}$ relation for inactive galaxies (Woo et al. 2010, 2013, 2015; Graham et al. 2011; Park et al. 2012a; van den Bosch et al. 2015; Shankar et al. 2016, 2019; Batiste et al. 2017a). However, these authors noted that the discrepancy between AGNs and inactive galaxies may be due to sample selection bias.

The Local Luminous AGNs with Matched Analogues (LLAMA) sample was created to overcome selection biases in the studies of local AGNs (Davies et al. 2015). The AGNs in this sample are selected in the ultra-hard X-rays, avoiding issues with obscuration for all but the most Compton-thick galaxies. As the name implies it comes with a sample of (stellar mass, distance, inclination, and Hubble type) matched inactive galaxies to be able to compare galaxy properties among AGNs and similar inactive host galaxies. Over the last five years, this sample has been observed with VLT/X-shooter, VLT/SINFONI, APEX and HST, and more observations are planned or proposed. These observations have so far been used to study the environmental dependence of AGN activity (Davies et al. 2017), nuclear stellar kinematics (Lin et al. 2018), the gas content and star formation efficiencies (Rosario et al. 2018), and the nuclear star formation histories (Burtscher et al., in prep.). In addition several singleobject studies have been performed with this rich data set, for 
example, on NGC 2110 (Rosario et al. 2019) and NGC 5728 (Shimizu et al. 2019).

In this paper, we present stellar velocity dispersions $\left(\sigma_{\star}\right)$ calculated from the $\mathrm{Ca}$ II triplet (CaT) and the CO (2-0) absorption features and the broad-line-based single-epoch black hole mass estimates for the hard X-ray selected LLAMA sample using the available X-shooter and SINFONI data. We present a comparison of our results with the $M_{\mathrm{BH}}-\sigma_{\star}$ plane. We aim to understand the physical properties of the LLAMA sample of AGNs, and we also aim to test the robustness of the parameters that are used for the AGN $M_{\mathrm{BH}}-\sigma \star$ relation. The paper is organized as follows: Section 2 reviews sample selection, observation, and data reduction processes. Section 3 describes our estimation methods and the tests we performed for studying the robustness of $M_{\mathrm{BH}}-\sigma_{\star}$ parameters. In Sect. 4 , we discuss our results. Finally, we conclude the paper in Sect. 5 .

\section{Sample selection, observation, and data reduction}

\subsection{Sample selection}

A complete volume-limited sample of the most luminous $\mathrm{X}$-ray-selected local AGNs in the southern hemisphere was compiled by Davies et al. (2015) as the LLAMA project. The AGN sample was selected from the Swift-BAT 58-month survey (Baumgartner et al. 2010) using the following three criteria:

1. High X-ray luminosity $\left(\log L_{14-195 \mathrm{keV}} \geq 42.5 \mathrm{erg} \mathrm{s}^{-1}\right)$, to select bona-fide AGNs

2. Low-redshift AGNs $(z<0.01)$ to spatially resolve the nuclear regions

3. Observable from VLT $\left(\delta<15^{\circ}\right)$

The LLAMA AGN sample comprises ten Type 1 and ten Type 2 AGNs (Davies et al. 2015). These AGNs were selected to be the most luminous local AGNs and are sufficiently powerful to sustain a BLR.

The matching inactive galaxy sample was selected by Davies et al. (2015) based on the following criteria: $H$-band luminosity (as a proxy of stellar mass), redshift, distance, inclination, and host galaxy morphology. Based on these criteria, 19 inactive galaxies comprise the LLAMA inactive galaxy sample.

In this work, we compare the physical properties of both sample. The mean $H$-band luminosities are $\log L_{\mathrm{H}}\left[L_{\odot}\right]=10.3 \pm 0.3$ for AGN sample and $\log L_{\mathrm{H}}\left[L_{\odot}\right]=10.2 \pm 0.4$ for inactive galaxy sample. The LLAMA inactive galaxies are also selected within the same redshift cutoff as active galaxy sample, which is $z<0.01$. The active and inactive galaxy samples have redshift-independent mean distances 31 and $24 \mathrm{Mpc}$, respectively. The average inclinations for each sample are found to be $\sim 45^{\circ}$. Both active and inactive samples have a wide variety of galaxy morphologies with a peak distribution around early-disk types ( $\mathrm{S} 0$ and $\mathrm{Sa}$ ). Finally, also the presence/absence of a bar is also matched for both samples where possible.

\subsection{Observations and data reduction}

The medium-resolution spectrograph X-shooter on the Very Large Telescope (VLT), covering $0.3-2.3 \mu \mathrm{m}$, was used to observe the LLAMA sample. The X-shooter observations were performed between November 2013 and June 2015, using the IFU-offset mode with a field of view (FOV) of 1 "! $8 \times 4^{\prime \prime}$ Spectroscopic standard star observations were performed on the same nights with similar atmospheric conditions, and telluric standard stars were observed before and after the target. Data were obtained with resolution $R \sim 8400,13200,8300$ for the ultraviolet (UVB), visual (VIS) and near-infrared (NIR) arms, respectively. The $\mathrm{X}$-shooter data cubes were obtained using the ESO X-shooter pipeline v2.6.0 (Modigliani et al. 2010) within the ESO Reflex environment (Freudling et al. 2013). Finally, the spectra were corrected for telluric absorption using telluric standard stars. The data analysis of the X-shooter observations was performed by Schnorr-Müller et al. (2016) and included most notably a correction for the [Fe II] multiplets in the 4000-5600 ̊ wavelength range. A more detailed description of the X-shooter data processing will be given in Burtscher et al. (in prep.).

The SINFONI observations were performed between 2014 April and 2018 March with the $\mathrm{H}+\mathrm{K}$ grating at a spectral resolution $R \sim 1500$ for each $00^{\prime \prime} .05 \times 00^{\prime \prime} .1$ spatial pixel leading to a total FOV of $3^{\prime \prime} 0 \times 3^{\prime \prime} 0$. The observations were performed in adaptive optics (AO) mode and a standard NIR nodding technique was used. The telluric standard stars were observed before and after the target observations to obtain similar atmospheric conditions. The SINFONI data were reduced using the SINFONI custom reduction package SPRED (Abuter et al. 2006). Further details about observation and data reduction are described by Lin et al. (2018).

We note that the majority of X-shooter and SINFONI observations were performed for both active and inactive galaxy sample and the same data reduction approach was used for them. In Table 1, we present the observation lists and basic properties of the LLAMA AGN and inactive galaxy sample.

\section{Methods and models}

We performed the spectral analysis for 20 AGNs in our sample. In the first step, the AGN continuum was modeled and extracted from the spectra using additive polynomials in the form of power-law functions. We fit the spectra of each AGN using stellar templates to determine stellar velocity dispersions (see Sect. 3.1). The resulting stellar velocity dispersion estimates are presented in Table 2. The emission lines from BLR and NLR were fit by applying multiple Gaussian models (Sect. 3.3). Finally, black hole masses were obtained through virial "singleepoch" empirical correlations (Sect. 3.4). The results are presented in Table 3.

\subsection{Velocity dispersion calculations}

We obtained stellar velocity dispersions from the Ca II triplet $(8498,8552,8662 \AA)$, where the AGN contamination is typically weaker than in the $\mathrm{Mg}$ b triplet $(5069,5154,5160 \AA$ ) (Greene \& Ho 2006b; Harris et al. 2012). We also estimated stellar velocity dispersions from the $\mathrm{CO}(2-0)$ absorption at $2.2935 \mu \mathrm{m}$, since it is less affected by dust extinction. Riffel et al. (2015) report that giant and super-giant stars are the dominant contributor for $\mathrm{CaT}$ and $\mathrm{CO}$ regions, respectively. To estimate stellar velocity dispersions, we used the penalized pixel-fitting (pPXF) method (Cappellari \& Emsellem 2004; Cappellari 2017) adopting the Xshooter G, M, K stellar population spectral library (127 stars) of Chen et al. (2014) to fit the CaT absorption lines and the GEMINI NIR stellar library with spectral types ranging from F7 III to M5 III (60 stars) (Winge et al. 2009) to fit the CO (2-0) absorption lines.

The pPXF method adopts the Gauss-Hermite parametrization for the line-of-sight velocity distribution in the pixel space, where bad pixels and emission lines can be easily excluded 
Table 1. Galaxy properties: X-shooter, and SINFONI observation lists of our sample of galaxies.

\begin{tabular}{|c|c|c|c|c|c|c|c|c|c|}
\hline \multicolumn{4}{|c|}{ Properties } & \multicolumn{3}{|l|}{ X-shooter } & \multicolumn{3}{|l|}{ SINFONI } \\
\hline Object name & $\begin{array}{l}\text { Dist } \\
(\mathrm{Mpc})\end{array}$ & Morph & $\begin{array}{c}\log L \\
\left(\operatorname{erg~s}^{-1}\right)\end{array}$ & $\begin{array}{c}\text { Obs. Date } \\
(\mathrm{DD} / \mathrm{MM} / \mathrm{YY})\end{array}$ & AirM & $\begin{array}{l}\text { Seeing } \\
\left({ }^{\prime \prime}\right)\end{array}$ & $\begin{array}{c}\text { Obs. Date } \\
\text { (DD/MM/YY) }\end{array}$ & AirM & $\begin{array}{c}\text { Seeing } \\
\left({ }^{\prime \prime}\right)\end{array}$ \\
\hline 1 & 2 & 3 & $4 \mathrm{~A}$ & 5 & 6 & 7 & 8 & 9 & 10 \\
\hline ESO 137-G034 & 35 & $\mathrm{~S} 0 \mathrm{a}(\mathrm{AB})$ & 42.76 & $19 / 05 / 19$ & 1.2 & 0.78 & $18 / 04 / 14$ & 1.2 & 0.75 \\
\hline ESO 021-G004 & 39 & $\mathrm{SA}(\mathrm{s}) 0 / \mathrm{a}$ & 42.70 & $02 / 08 / 16$ & 1.8 & 0.83 & - & - & - \\
\hline MCG-05-14-12 & 41 & S0 & 42.65 & $11 / 12 / 13$ & 1.0 & 0.61 & - & - & - \\
\hline MCG-05-23-16 & 35 & So & 43.50 & $22 / 01 / 14$ & 1.1 & 1.21 & $14 / 01 / 17$ & 1.1 & 1.00 \\
\hline MCG-06-30-15 & 27 & $\mathrm{~S} ?$ & 42.91 & $16 / 01 / 15$ & 1.1 & 0.83 & $04 / 06 / 14$ & 1.1 & 1.08 \\
\hline NCG 1365 & 18 & $\mathrm{Sb}(\mathrm{B})$ & 42.60 & $10 / 12 / 13$ & 1.0 & 1.34 & $18 / 11 / 10$ & 1.1 & 0.78 \\
\hline NGC 2110 & 27 & $\mathrm{~S} ?(\mathrm{AB})$ & 43.63 & $16 / 01 / 15$ & 1.1 & 0.59 & $15 / 01 / 11$ & 1.1 & 0.83 \\
\hline NGC 2992 & 36 & $\mathrm{Sa}$ & 42.52 & $26 / 02 / 14$ & 1.3 & 0.72 & $05 / 02 / 17$ & 1.0 & 0.85 \\
\hline NGC 3081 & 34 & $(\mathrm{R}) \mathrm{SAB}(\mathrm{r}) 0 / \mathrm{a}$ & 43.29 & $20 / 02 / 14$ & 1.2 & 0.82 & $14 / 03 / 17$ & 1.2 & 0.76 \\
\hline NGC 3783 & 38 & $\mathrm{Sb}(\mathrm{B})$ & 43.58 & $11 / 03 / 14$ & 1.4 & 0.81 & $16 / 02 / 15$ & 1.2 & 1.04 \\
\hline NGC 4235 & 37 & $\mathrm{Sa}$ & 42.64 & $13 / 05 / 15$ & 1.2 & 0.73 & - & - & - \\
\hline NGC 4388 & 39 & $\mathrm{SA}(\mathrm{s}) \mathrm{b}(\mathrm{B})$ & 43.70 & - & - & - & $24 / 02 / 15$ & 1.5 & 0.35 \\
\hline NGC 4593 & 37 & $\mathrm{Sb}(\mathrm{B})$ & 43.20 & $10 / 03 / 14$ & 1.3 & 0.80 & $23 / 01 / 15$ & 1.1 & 0.88 \\
\hline NGC 5128 & 3.8 & So p & 43.02 & $21 / 05 / 15$ & 1.1 & 0.76 & - & - & - \\
\hline NGC 5506 & 27 & Sa p & 43.30 & $03 / 03 / 16$ & 1.1 & 0.64 & $12 / 03 / 15$ & 1.098 & 0.72 \\
\hline NGC 5728 & 39 & SAB(r)a: & 43.36 & $13 / 05 / 15$ & 1.0 & 0.81 & $25 / 06 / 15$ & 1.3 & 0.75 \\
\hline NGC 6814 & 23 & $\mathrm{SAB}(\mathrm{rs}) \mathrm{bc}$ & 42.75 & $13 / 05 / 15$ & 1.1 & 0.86 & $05 / 06 / 14$ & 1.0 & 0.83 \\
\hline NGC 7172 & 37 & $\mathrm{Sa}$ & 43.32 & $12 / 08 / 15$ & 1.0 & 1.6 & $20 / 07 / 14$ & 1.0 & 0.77 \\
\hline NGC 7213 & 22 & $\mathrm{Sa}(\mathrm{s})$ & 42.49 & $13 / 07 / 16$ & 1.3 & 0.47 & $16 / 07 / 14$ & 1.1 & 0.83 \\
\hline NGC 7582 & 22 & $\left(\mathrm{R}^{\prime}\right) \mathrm{SB}(\mathrm{s}) \mathrm{ab}(\mathrm{B})$ & 43.29 & $27 / 07 / 17$ & 1.2 & 0.69 & $14 / 07 / 14$ & 1.1 & 0.91 \\
\hline 1 & 2 & 3 & 4B & 5 & 6 & 7 & 8 & 9 & 10 \\
\hline ESO 093-G003 & 22 & $\mathrm{SAB}(\mathrm{r}) 0 / \mathrm{a} ?$ & 9.86 & $22 / 01 / 14$ & 1.3 & 0.98 & $06 / 04 / 17$ & 1.4 & 0.86 \\
\hline ESO 208-G021 & 17 & SABO & 10.88 & $12 / 12 / 13$ & 1.1 & 0.95 & $14 / 03 / 17$ & 1.2 & 1.02 \\
\hline NGC 718 & 23 & $\mathrm{SAB}(\mathrm{s}) \mathrm{a}$ & 9.89 & $05 / 12 / 15$ & 1.2 & 0.61 & $13 / 08 / 14$ & 1.2 & 0.82 \\
\hline NGC 1079 & 19 & (R)SAB(rs)0/a & 9.91 & $23 / 11 / 13$ & 1.0 & 1.12 & $17 / 11 / 051$ & 1.1 & 0.88 \\
\hline NGC 1315 & 21 & SB0? & 10.07 & $11 / 12 / 13$ & 1.0 & 0.83 & - & - & - \\
\hline NGC 1947 & 19 & S0 p & 10.45 & $23 / 12 / 13$ & 1.4 & 0.77 & - & - & - \\
\hline NGC 2775 & 21 & $\mathrm{SA}(\mathrm{r}) \mathrm{ab}$ & 9.84 & $15 / 11 / 15$ & 1.5 & 0.74 & - & - & - \\
\hline NGC 3175 & 14 & $\mathrm{SAB}(\mathrm{s}) \mathrm{a} ?$ & 10.07 & $09 / 03 / 14$ & 1.2 & 1.13 & 06/04/17 & 1.0 & 0.88 \\
\hline NGC 3351 & 11 & $\mathrm{SB}(\mathrm{r}) \mathrm{b}$ & 10.39 & $21 / 02 / 14$ & 1.3 & 1.04 & $27 / 01 / 15$ & 1.3 & 0.89 \\
\hline NGC 3717 & 24 & $\mathrm{SAb}$ & 10.40 & $22 / 03 / 14$ & 1.2 & 1.34 & - & - & - \\
\hline NGC 3749 & 42 & $\mathrm{SA}(\mathrm{s}) \mathrm{a}$ & 10.48 & $22 / 03 / 14$ & 1.0 & 0.93 & - & - & - \\
\hline NGC 4224 & 41 & $\mathrm{SA}(\mathrm{s}) \mathrm{a}$ & 10.22 & $13 / 05 / 15$ & 1.2 & 0.66 & $24 / 02 / 15$ & 1.2 & 0.91 \\
\hline NGC 4254 & 15 & $\mathrm{SA}(\mathrm{s}) \mathrm{c}$ & 10.22 & $02 / 06 / 16$ & 1.3 & 0.77 & $09 / 03 / 15$ & 1.5 & 0.84 \\
\hline NGC 4260 & 31 & $\mathrm{SB}(\mathrm{s}) \mathrm{a}$ & 10.25 & - & - & - & - & - & - \\
\hline NGC 5037 & 35 & $\mathrm{SA}(\mathrm{s}) \mathrm{a}$ & 10.30 & $13 / 05 / 15$ & 1.0 & 0.70 & - & - & - \\
\hline NGC 5845 & 25 & $\mathrm{E}$ & 10.46 & $16 / 03 / 16$ & 1.2 & 0.69 & $14 / 03 / 17$ & 1.2 & 0.61 \\
\hline NGC 5921 & 21 & $\mathrm{SB}(\mathrm{r}) \mathrm{bc}$ & 10.08 & $16 / 06 / 15$ & 1.2 & 0.71 & - & - & - \\
\hline NGC 7727 & 26 & $\mathrm{SAB}(\mathrm{s}) \mathrm{a} \mathrm{p}$ & 10.41 & $25 / 08 / 15$ & 1.0 & 0.68 & 21/07/14 & 1.0 & 0.89 \\
\hline IC 4653 & 26 & $\mathrm{SB} 0 / \mathrm{a}(\mathrm{r}) \mathrm{p}$ & 9.48 & $19 / 05 / 2015$ & 1.2 & 0.79 & $25 / 07 / 2017$ & 1.6 & 1.11 \\
\hline
\end{tabular}

Notes. Sector 1 (top): the LLAMA AGNs; Sector 2 (bottom): the LLAMA inactive galaxies. (1) Object name, (2) distance, (3) Galaxy morphology, (4) (a) logarithmic X-ray luminosity, (b) integrated $H$-band luminosity in logarithm in solar unit, (5) X-shooter observation date, (6) air mass during the observation, (7) seeing, (8) SINFONI observation date, (9) air mass during the observation, and (10) seeing. Galaxy morphologies and distances are taken from the NASA Extragalactic database. B and AB indicates the existence and absence of bar, respectively. The hard X-ray luminosities (14-195 keV) are taken from the Swift-BAT 70 months survey (Baumgartner et al. 2010), where X-ray luminosities were corrected for absorption based on X-ray fittings by Ricci et al. (2017a). The list of abbreviations: distance (Dist) observation (Obs), morphology (Morph), air mass (AirM), and peculiar (p). Seyfert types of the LLAMA AGNs are presented in Table 2.

from the spectra, and continuum matching can be performed directly using additive polynomials. The pPXF measures stellar velocity dispersions by making initial guesses using a broadening function for stellar templates. The fit parameters $(V, \sigma$, $h_{3}, \ldots, h_{\mathrm{m}}$ ), where $h_{i}$ is the Hermite polynomial for the $i$ th parameter, are fitted simultaneously using pPXF, but it adds an adjustable penalty term to the $\chi^{2}$ to optimize the fit. In this way, the best-fitting parameters of the Gauss-Hermite series can be estimated and the lowest $\chi^{2}$ are provided by the definition of this method (e.g., van der Marel \& Franx 1993, and references therein). The uncertainties of stellar velocity dispersion estimates were obtained via bootstrapping by randomly resampling the residuals of the best fit of pPXF, and repeating pPXF fitting 100 times. 
T. Caglar et al.: LLAMA: The $M_{\mathrm{BH}}-\sigma_{\star}$ relation of the most luminous local AGNs

Table 2. Stellar velocity dispersion comparison between the estimates from CaT and CO (2-0) absorption lines.

\begin{tabular}{|c|c|c|c|c|c|c|c|c|}
\hline Object & $\begin{array}{c}r_{\mathrm{e}} \\
(\operatorname{arcsec})\end{array}$ & $\begin{array}{c}\sigma \star \mathrm{CaT} \\
\left(\mathrm{km} \mathrm{s}^{-1}\right)\end{array}$ & $\begin{array}{l}\sigma_{\star \mathrm{CO}_{(2-0)}} \\
\left(\mathrm{km} \mathrm{s}^{-1}\right) \\
\end{array}$ & $\begin{array}{c}\text { Correction } \\
(\%)\end{array}$ & Sérsic index & $\mathrm{B} / \mathrm{T}$ & Bulge type & Seyfert activity \\
\hline 1 & 2 & 3 & 4 & 5 & 6 & 7 & 8 & \\
\hline ESO 137-G034 & 6.94 (a) & $128 \pm 4$ & $130 \pm 7$ & $10^{(\star)}$ & 2.13 (a) & 0.22 (a) & PB? & Sy 2 (II) \\
\hline ESO 021-G004 & $16.7(\mathrm{r})$ & $178 \pm 3$ & - & $10^{(\star)}$ & - & - & - & Sy $2(0)$ \\
\hline MCG-05-14-12 & $4.41(\mathrm{r})$ & $73 \pm 5$ & - & $10^{(\star)}$ & - & - & - & Sy $1.0(0 n)$ \\
\hline MCG-05-23-16 & 9.37 (c) & $135 \pm 4$ & $140 \pm 7$ & 11.8 & $3.20(\mathrm{c})$ & - & $\mathrm{CB} ?$ & Sy 1.9 (I) \\
\hline MCG-06-30-15 & $0.63(d)$ & $95 \pm 5$ & $101 \pm 6$ & $10^{(\star)}$ & $1.29(\mathrm{~d})$ & $0.06(\mathrm{~d})$ & PB & Sy $1.2(0 n)$ \\
\hline NGC 1365 & $12.8(\mathrm{e})$ & $121 \pm 5$ & $120 \pm 6$ & 20 & $0.86(\mathrm{e})$ & $0.25(\mathrm{e})$ & PB & Sy 1.8 (I) \\
\hline NGC 2110 & $6.80(f)$ & $227 \pm 3$ & $231 \pm 5$ & $10^{(\star)}$ & $2.70(\mathrm{f})$ & 0.39 (f) & $\mathrm{CB}$ & Sy 2 (II) \\
\hline NGC 2992 & $14.2(\mathrm{r})$ & $154 \pm 3$ & $156 \pm 5$ & 12.2 & - & - & - & Sy $1.8(\mathrm{I})$ \\
\hline NGC 3081 & $1.34(\mathrm{~g})$ & $132 \pm 4$ & $135 \pm 7$ & $10^{(\star)}$ & $2.10(\mathrm{~g})$ & $0.10(\mathrm{~g})$ & PB? & Sy 2(II) \\
\hline NGC 3783 & 1.45 (a) & $125 \pm 5$ & $134 \pm 8$ & $10^{(\star)}$ & 1.24 (a) & 0.21 (a) & PB & Sy 1.2 (I) \\
\hline NGC 4235 & $2.70(\mathrm{o})$ & $142 \pm 5$ & - & $10^{(\star)}$ & $6.00(\mathrm{~h})$ & 0.50 (i) & $\mathrm{CB}$ & Sy 1.2 (I) \\
\hline NGC 4388 & $5.62(\mathrm{p})$ & - & $117 \pm 6$ & 18.8 & $0.50(\mathrm{j})$ & - & - & Sy 2 (II) \\
\hline NGC 4593 & $6.21(\mathrm{~b})$ & $139 \pm 5$ & $145 \pm 4$ & 1.4 & $1.37(b)$ & 0.18 (b) & PB & Sy $1.2(1)$ \\
\hline NGC 5128 & $8.62(\mathrm{k})$ & $199 \pm 8$ & - & $10^{(\star)}$ & $2.63(\mathrm{k})$ & $1.00(1)$ & $\mathrm{CB}$ & Sy 2 (III) \\
\hline NGC 5506 & $2.06(\mathrm{~m})$ & - & $118 \pm 47$ & $10^{(\star)}$ & $0.50(\mathrm{~m})$ & $0.06(\mathrm{~m})$ & $\mathrm{PB}$ & Sy $1 \mathrm{i}$ (IV) \\
\hline NGC 5728 & 4.02 (a) & $168 \pm 7$ & $169 \pm 9$ & 2.8 & 1.10 (a) & 0.23 (a) & PB? & Sy 2 (II) \\
\hline NGC 6814 & $1.08(\mathrm{a})$ & $99 \pm 4$ & $110 \pm 4$ & 0 & $1.08(\mathrm{a})$ & 0.09 (a) & PB & Sy $1.2(\mathrm{I})$ \\
\hline NGC 7172 & 1.16 (a) & $145 \pm 5$ & $146 \pm 6$ & $10^{(\star)}$ & $1.16(a)$ & 0.25 (a) & PB? & Sy 2 (II) \\
\hline NGC 7213 & 13.7 (a) & $209 \pm 7$ & $211 \pm 10$ & 0 & 2.57 (a) & 0.70 (a) & $\mathrm{CB}$ & Sy $1.0(\mathrm{~V} L)$ \\
\hline NGC 7582 & 1.99 (a) & $129 \pm 4$ & $130 \pm 6$ & $10^{(\star)}$ & $2.72(\mathrm{a})$ & 0.28 (a) & PB? & Sy 2 (II) \\
\hline ESO 093-G003 & $11.5(\mathrm{r})$ & $87 \pm 5$ & $85 \pm 8$ & - & - & - & - & - \\
\hline ESO 208-G021 & $7.47(\mathrm{~g})$ & $214 \pm 6$ & $213 \pm 9$ & - & $4.20(\mathrm{~g})$ & $0.97(\mathrm{~g})$ & $\mathrm{CB}$ & - \\
\hline NGC 718 & 2.09 (a) & $104 \pm 5$ & $118 \pm 7$ & - & 1.32 (a) & 0.28 (a) & PB & - \\
\hline NGC 1079 & $4.94(\mathrm{~g})$ & $114 \pm 2$ & $123 \pm 7$ & - & $2.20(\mathrm{~g})$ & $0.25(\mathrm{~g})$ & PB? & - \\
\hline NGC 1315 & $16.1(\mathrm{r})$ & $77 \pm 3$ & - & - & - & - & - & \\
\hline NGC 1947 & 30.1 (b) & $147 \pm 3$ & - & - & $2.51(\mathrm{~b})$ & 0.68 (b) & $\mathrm{CB}$ & - \\
\hline NGC 2775 & $63.2(\mathrm{~h})$ & $175 \pm 6$ & - & - & 3.49 (h) & 0.75 (i) & $\mathrm{CB}$ & - \\
\hline NGC 3175 & $40.1(\mathrm{r})$ & $73 \pm 5$ & $72 \pm 7$ & - & - & - & - & - \\
\hline NGC 3351 & 6.95 (a) & $91 \pm 4$ & $91 \pm 7$ & - & $0.80(a)$ & 0.22 (a) & PB & - \\
\hline NGC 3717 & $32.5(\mathrm{r})$ & $137 \pm 5$ & - & - & - & - & - & - \\
\hline NGC 4224 & 5.01 (a) & $146 \pm 3$ & $145 \pm 8$ & - & $2.53(\mathrm{a})$ & 0.29 (a) & $\mathrm{CB} ?$ & - \\
\hline NGC 4254 & 12.59 (a) & $82 \pm 5$ & $87 \pm 7$ & - & 1.99 (a) & 0.19 (a) & PB? & - \\
\hline NGC 5037 & $23.2(\mathrm{r})$ & $168 \pm 3$ & - & - & - & - & - & - \\
\hline NGC 5845 & $0.49(\mathrm{p})$ & $262 \pm 6$ & $267 \pm 10$ & - & - & 1.0 (i) & $\mathrm{CB}$ & - \\
\hline NGC 5921 & $3.59(\mathrm{n})$ & $80 \pm 2$ & - & - & $1.60(\mathrm{n})$ & 0.50 (i) & PB? & - \\
\hline NGC 7727 & 5.07 (a) & $201 \pm 5$ & $199 \pm 7$ & - & 1.68 (a) & 0.36 (a) & CB? & - \\
\hline IC 4653 & $17.0(\mathrm{r})$ & $64 \pm 5$ & - & - & - & - & - & - \\
\hline
\end{tabular}

Notes. Sector 1 (top): LLAMA AGNs; Sector 2 (bottom): the LLAMA inactive Galaxies. Columns are from left to right as follows: (1) object name; (2) bulge effective radius; (3) stellar velocity dispersion estimates from the CaT absorption lines; (4) stellar velocity dispersion estimates from the $\mathrm{CO}(2-0)$ transmission; (5) rotation contribution in percentage; $\left.{ }^{\star}{ }^{\star}\right)$ the assumed rotation contribution, which is the average rotation contribution of LLAMA sample; (6) Sérsic index; (7) bulge-to-total mass ratio (B/T); and (8) bulge type, where PB and CB refer to pseudo-bulge and classicalbulge, respectively. The CaT region of NGC 5506 is highly contaminated by AGN emission lines, therefore the $\sigma_{\mathrm{CaT}}$ is not presented in our study. There is no available X-shooter observation for NGC 4388, but there are three available $\sigma_{\star \text { CaT }}$ estimates from the literature. The reported $\sigma_{\star \text { CaT }}$ values differ significantly: $\sigma_{\star \mathrm{CaT}}=119 \mathrm{~km} \mathrm{~s}^{-1}$ (Terlevich et al. 1990), $\sigma_{\star \mathrm{CaT}}=165 \pm 21 \mathrm{~km} \mathrm{~s}^{-1}$ (Riffel et al. 2015), and $\sigma_{\star \mathrm{CaT}}=76 \mathrm{~km} \mathrm{~s}^{-1}(\mathrm{Greene}$ et al. 2010). But the central velocity dispersion measurements of this galaxy, as reported by Greene et al. (2010), Saglia et al. (2016), van den Bosch (2016), are in the range $\sim 100-120 \mathrm{~km} \mathrm{~s}^{-1}$, which are consistent with our $\sigma_{\mathrm{CO}(2-0)}$ estimate. Therefore, we used our $\sigma_{\mathrm{CO}(2-0)}$ estimate as a surrogate for $\sigma_{\star \mathrm{CaT}}$ for NGC 4388 .

References. Reference for Seyfert activity in the literature; (0): this work, (I): Schnorr-Müller et al. (2016), (II): Gu et al. (2006), (III): Maiolino \& Rieke (2013) (IV): Véron \& Véron (2010), (V): González-Martín et al. (2015). We note that $L$ is LINER and $n$ indicates narrow-line Seyfert 1 galaxies according to our spectral investigations. Bulge properties are taken from as follows: (a) Lin et al. (2018), (b) Gao et al. (2019), (c) Capetti (2007), (d) Hu et al. (2016), (e) Combes et al. (2019), (f) Gadotti (2008), (g) Laurikainen et al. (2010), (h) Salo et al. (2015), (i) de Lapparent et al. (2011), (j) Greene et al. (2010), (k) Fisher \& Drory (2010), (l) Kormendy et al. (2015), (m) Yoshino \& Yamauchi (2015), (n) Knapen et al. (2003), (o) Baggett et al. (1998), (p) van den Bosch (2016), (r) Skrutskie et al. (2006).

To match the spectral resolutions of galaxy and template spectra, the template spectra were convolved with the line spread function of $\sim 70 \mathrm{~km} \mathrm{~s}^{-1}$ for SINFONI data, while the XSHOOTER template spectra were convolved by $\sim 5 \mathrm{~km} \mathrm{~s}^{-1}$.
Since the CO absorption lines in the NIR tend to have lower signal-to-noise ratio $(S / N \sim 10)$ relative to the CaT absorption lines $(S / N \sim 50)$, we did not use $h_{3}$ and $h_{4}$ higher order moments for the $\mathrm{CO}$ (2-0) absorption lines fitting. The fitting procedure 
Table 3. Spectral results of the LLAMA AGN sample.

\begin{tabular}{|c|c|c|c|c|c|c|c|c|}
\hline 1 & 2 & 3 & 4 & $5 \mathrm{~A}$ & 6 & 7 & 8 & 9 \\
\hline Object & $\begin{array}{c}A_{V}(\mathrm{BLR}) \\
(\mathrm{mag})\end{array}$ & $\begin{array}{c}F W H M(\mathrm{H} \alpha) \\
1^{\prime \prime} .8 \times 4^{\prime \prime} \\
\left(\mathrm{km} \mathrm{s}^{-1}\right)\end{array}$ & $\begin{array}{c}F W H M(\mathrm{H} \beta) \\
1^{\prime \prime} 8 \times 4^{\prime \prime} \\
\left(\mathrm{km} \mathrm{s}^{-1}\right)\end{array}$ & $\begin{array}{c}M_{\mathrm{BH}}(F W H M) \\
(\mathrm{H} \alpha) \\
\left(10^{6} M_{\odot}\right)\end{array}$ & $\begin{array}{c}M_{\mathrm{BH}}\left(\sigma_{\text {Line }}\right) \\
(\mathrm{H} \beta) \\
\left(10^{6} M_{\odot}\right)\end{array}$ & $\begin{array}{c}\dot{M} \\
\left(10^{-2} M_{\odot} \mathrm{yr}^{-1}\right)\end{array}$ & $\lambda_{\text {Edd }}$ & $\begin{array}{l}\Delta M \\
(\operatorname{dex}) \\
\end{array}$ \\
\hline MCG-05-14-12 & $0.0 \pm 0.2$ & $1836.0 \pm 119$ & $2019.1 \pm 167$ & $2.29 \pm 0.68$ & $2.30 \pm 1.38$ & 6.12 & 0.120 & 0.23 \\
\hline MCG-06-30-15 & $2.8 \pm 0.4$ & $1456.8 \pm 122$ & $1588.4 \pm 198$ & $7.38 \pm 1.98$ & $5.97 \pm 1.92$ & 12.0 & 0.073 & -0.06 \\
\hline NGC 3783 & $0.1 \pm 0.2$ & $3002.3 \pm 196$ & $3102.3 \pm 312$ & $11.2 \pm 3.61$ & $10.1 \pm 4.72$ & 67.3 & 0.272 & -0.27 \\
\hline NGC 4235 & $1.5 \pm 0.5$ & $6611.1 \pm 461$ & - & $55.8 \pm 15.9$ & - & 5.96 & 0.005 & 0.27 \\
\hline NGC 4593 & $0.0 \pm 0.1$ & $3741.8 \pm 213$ & $4179.4 \pm 294$ & $12.4 \pm 3.91$ & $10.0 \pm 4.38$ & 25.3 & 0.091 & -0.50 \\
\hline NGC 6814 & $0.4 \pm 0.4$ & $3299.3 \pm 191$ & $3771.0 \pm 279$ & $11.6 \pm 3.67$ & $13.4 \pm 4.12$ & 7.92 & 0.031 & -0.16 \\
\hline NGC 7213 & $0.0 \pm 0.3^{(\bullet)}$ & $2732.8 \pm 264$ & $3302.0 \pm 701$ & $6.46 \pm 2.01$ & $6.45 \pm 2.47$ & 4.05 & 0.028 & -1.46 \\
\hline Object & $\begin{array}{c}A_{V}(\mathrm{BLR}) \\
(\mathrm{mag})\end{array}$ & $\begin{array}{c}F W H M(\mathrm{H} \alpha) \\
1^{\prime \prime} 8 \times 4^{\prime \prime} \\
\left(\mathrm{km} \mathrm{s}^{-1}\right) \\
\end{array}$ & $\begin{array}{c}F W H M(\mathrm{~Pa} \beta) \\
1^{\prime \prime} .8 \times 4^{\prime \prime} \\
\left(\mathrm{km} \mathrm{s}^{-1}\right) \\
\end{array}$ & $\begin{array}{c}M_{\mathrm{BH}}(F W H M) \\
(\mathrm{H} \alpha) \\
\left(10^{6} M_{\odot}\right)\end{array}$ & $\begin{array}{c}M_{\mathrm{BH}}(F W H M) \\
(\mathrm{Pa} \beta) \\
\left(10^{6} M_{\odot}\right)\end{array}$ & $\begin{array}{c}\dot{M} \\
\left(10^{-2} M_{\odot} \mathrm{yr}^{-1}\right)\end{array}$ & $\lambda_{\text {Edd }}$ & $\begin{array}{l}\Delta M \\
(\operatorname{dex}) \\
\end{array}$ \\
\hline MCG-05-23-16 & $4.2 \pm 0.9$ & $2186.1 \pm 166$ & $1935.4 \pm 196$ & $27.1 \pm 8.74$ & $25.3 \pm 8.84$ & 54.8 & 0.091 & 0.17 \\
\hline NGC 1365 & $4.4 \pm 0.9$ & $2406.1 \pm 180$ & $1872.0 \pm 352$ & $19.7 \pm 5.77$ & $13.8 \pm 5.96$ & 5.38 & 0.012 & -0.48 \\
\hline NGC 2992 & $4.5 \pm 0.8$ & $2085.5 \pm 189$ & $2180.9 \pm 260$ & $22.8 \pm 6.74$ & $26.4 \pm 9.02$ & 4.38 & 0.004 & -0.10 \\
\hline 1 & 2 & 3 & 4 & $5 \mathrm{~B}$ & 6 & 7 & 8 & 9 \\
\hline Object & & & & $\begin{array}{c}M_{\mathrm{BH}} \\
\text { (Megamaser) } \\
\left(10^{6} M_{\odot}\right)\end{array}$ & & $\begin{array}{c}\dot{M} \\
\left(10^{-2} M_{\odot} \mathrm{yr}^{-1}\right)\end{array}$ & $\lambda_{\text {Edd }}$ & $\begin{array}{l}\Delta M \\
(\mathrm{dex})\end{array}$ \\
\hline NGC 4388 & - & - & - & $8.40 \pm 0.2^{(\bullet)}$ & - & 91.8 & 0.489 & -0.24 \\
\hline NGC 5728 & - & - & - & $23.0 \pm 2.3^{(\star)}$ & - & 38.2 & 0.074 & -0.42 \\
\hline 1 & 2 & 3 & 4 & $5 \mathrm{C}$ & 6 & 7 & 8 & 9 \\
\hline Object & & & & $\begin{array}{c}M_{\mathrm{BH}} \\
\left(M_{\mathrm{BH}}-\sigma_{\star}\right) \\
\left(10^{6} M_{\odot}\right) \\
\end{array}$ & & $\begin{array}{c}\dot{M} \\
\left(10^{-2} M_{\odot} \mathrm{yr}^{-1}\right) \\
\end{array}$ & $\lambda_{\text {Edd }}$ & $\begin{array}{l}\Delta M \\
(\operatorname{dex}) \\
\end{array}$ \\
\hline ESO 137-G034 & - & - & - & $21.5 \pm 15.8$ & - & 8.12 & 0.017 & 0.20 \\
\hline ESO 021-G004 & - & - & - & $52.1 \pm 38.4$ & - & 6.96 & 0.006 & 0.08 \\
\hline NGC 2110 & - & - & - & $150 \pm 110$ & - & 76.6 & 0.023 & -0.05 \\
\hline NGC 3081 & - & - & - & $36.6 \pm 26.9$ & - & 31.9 & 0.039 & 0.13 \\
\hline NGC 5128 & - & - & - & $66.3 \pm 48.9$ & - & 15.9 & 0.011 & 0.05 \\
\hline NGC 5506 & - & - & - & $22.4 \pm 17.2$ & - & 32.7 & 0.065 & 0.19 \\
\hline NGC 7172 & - & - & - & $53.4 \pm 39.3$ & - & 34.4 & 0.029 & 0.08 \\
\hline NGC 7582 & - & - & - & $30.5 \pm 22.4$ & - & 31.9 & 0.047 & 0.15 \\
\hline
\end{tabular}

Notes. Columns are from left to right as follows: (1) object names; (2) extinction values in the BLR are taken from Schnorr-Müller et al. (2016), and ${ }^{(\bullet)}$ extinction values in the BLR are estimated in this study using the same method provided by Schnorr-Müller et al. (2016); (3) FWHMs of H $\alpha$ emission line; (4) FWHMs of $\mathrm{H} \beta$ (or $\mathrm{Pa} \beta$ ) emission line; (5) black hole masses estimated from the following methods (for the different sections of the table) from top to bottom: (5A) $\mathrm{H} \alpha-$ FWHM (extinction-corrected), and (5B) megamaser disk, (5C) LLAMA $M_{\mathrm{BH}}-\sigma_{\star}$; (6) extinctioncorrected black hole masses estimated from the $\mathrm{H} \beta-\sigma_{\text {Line }}$ (or Pa $\beta-$ FWHM); (7) accretion rates; (8) Eddington ratios; and (9) offset from the $M_{\mathrm{BH}}-\sigma_{\star}$ relation of KH13 for given $\sigma_{\star}$. The first section of the table lists LLAMA Seyfert (Sy) 1-1.5 AGNs, the second section lists the three LLAMA Seyfert 1.8 and 1.9 AGNs, the third section lists the two LLAMA Seyfert 2 galaxies for which megamaser observations are available, and the fourth section lists the rest LLAMA Seyfert 2 galaxies for which $M_{\mathrm{BH}}$ are estimated from the LLAMA $M_{\mathrm{BH}}-\sigma_{\star}$ relation. NGC 5128 also has black hole mass estimates from the other methods: $M_{\mathrm{BH}} 4.5_{-1.0}^{+1.7} 10^{7} M_{\odot}$ from $\mathrm{H}_{2}$ gas kinematics by Neumayer $(2010), M_{\mathrm{BH}}=5.5 \pm 3.010^{7} M_{\odot}$ from stellar kinematics by Cappellari et al. (2009). We emphasize that our $M_{\mathrm{BH}}$ estimate for NGC 5128 is consistent with these results. The $\sigma_{\mathrm{CO}(2-0)}$ is used to obtain $M_{\mathrm{BH}}$ for NGC 5506 owing to the absence of $\sigma_{\mathrm{CaT}}$. The $M_{\mathrm{BH}}$ estimates from ${ }^{(\star)}$ Braatz et al. (2015), ${ }^{(\bullet)} \mathrm{Greene}$ et al. (2016). We note that we adopted $10 \%$ uncertainty for the $M_{\mathrm{BH}}$ of NGC 5728 owing to the absence of uncertainty in the related study. We adopted the $A_{V}$ (BLR) estimates obtained from the He II line ratios for MCG-05-23-16, NGC 1365 and NGC 2992 reported by Schnorr-Müller et al. (2016), since this method gives better results for Sy 1.8 and Sy 1.9 galaxies.

for the $\mathrm{CO}(2-0)$ absorption is explained in detail by Lin et al. (2018). We note that the AGN emission lines (e.g., O I $4998 \AA$, Fe II $8616 \AA$ ) are masked to increase the accuracy of stellar velocity dispersion calculations. We fit the integrated spectrum from the $\mathrm{X}$-shooter within 1 '! $8 \times 1$ 1".8 radius for CaT, whereas the integrated spectrum withing $3{ }^{\prime \prime} 0 \times 33^{\prime \prime}$. 0 radius was used for fitting CO (2-0). Finally, the resulting $\sigma_{\star}$ estimates were corrected for the instrumental broadening.
We then corrected $\sigma_{\star}$ estimates from the 1'.8 slit width to an effective radius using the following power-law function in the form:

$\sigma_{\text {re }}=\sigma_{\text {ap }}\left(\frac{r_{\text {ap }}}{r_{\text {re }}}\right)^{\alpha}$

where $\alpha$ is the slope, $r_{\mathrm{e}}$ is effective radius. Since $\log L_{\mathrm{H}}\left[L_{\odot}\right]=10.3 \pm 0.3$, which is assumed to be a proxy of stellar 
mass, for the LLAMA AGN sample we adopt $\alpha=0.077 \pm 0.012$ for late-type galaxies within $10<\log M_{\star}<11 M_{\odot}$ (FalcónBarroso et al. 2017). We note that we only present the resulting best-fitting $\sigma_{\star}$ values obtained within instrument aperture in Table 2. But, we note that effective radius-corrected $\sigma_{\star}$ values are used in our $M_{\mathrm{BH}}-\sigma_{\star}$ relation investigations. We note that the effective radius correction changes the LLAMA $\sigma_{\star}$ estimates from $2 \%$ to $18 \%$ with a mean of $\sim 10 \%$.

\subsection{Bulge properties of the LLAMA sample}

In this paragraph, we explain our method to identify the bulge properties of the LLAMA sample. Fisher \& Drory (2016) list a few major indicators for identifying pseudo-bulges. However, none of these diagnostics can be used alone to identify pseudobulges. In the same work, the authors also claim that pseudobulge hosting galaxies tend to have a Sérsic index $n<2$, bulge-to-total mass ratio $B / T \leq 0.35$ and $\sigma_{\star}<130 \mathrm{~km} \mathrm{~s}^{-1}$. Even though there are some exceptional cases, these three diagnostics are the best indicators for pseudo-bulges. Correspondingly, we collected $n$ and $B / T$ estimates from the literature. The collected diagnostic bulge-type indicators are presented in Table 2. These diagnostic parameters for pseudo-bulge identification demonstrate that the majority of the LLAMA AGN sample hosts pseudo-bulges $(\sim 65 \%)$.

\subsection{Emission line fitting}

We fit the spectra of our sample by adopting Astropy fitting routines (Astropy Collaboration 2013, 2018). The broad-line emission can often be fit sufficiently well using a single Gaussian profile, but sometimes more complex approaches are required (e.g., double-peak BLR emissions, extended wings; Peterson et al. 2004; Storchi-Bergmann et al. 2017). The $\mathrm{H} \beta$ profiles were fit within a rest-frame range $4700-5100 \AA$, whereas the $\mathrm{H} \alpha$ profiles were fit within a rest-frame range $6400-6800 \AA$. First, the AGN continuum of each AGN was modeled using a power-law function for $\mathrm{H} \beta, \mathrm{H} \alpha$ and $\mathrm{Pa} \beta$ region. We then describe narrow emission lines using single Gaussian profile for each AGN. For $\mathrm{H} \beta$ spectral region, we fit narrow $\mathrm{H} \beta$, [O III] (4959 $\AA$ ), and [O III] (5007 $\AA$ ) lines using single Gaussian profile for each narrow component. For $\mathrm{H} \alpha$ region, we fit narrow $\mathrm{H} \alpha$, [N II] (6548 $\AA$ ), [N II] $(6583 \AA)$, [S II] (6718.3 ̊), and [S II] (6732.7 $\AA$ ) lines using single Gaussian profile for each narrow component. However, since $\mathrm{H} \alpha$ is blended with two [N II] lines (6548 and $6583 \AA$ ), we adopted $F_{[\mathrm{N} \mathrm{II}]}^{6583 \AA}=2.96 \times F_{[\mathrm{NII}]}^{6548 \AA}$ (Osterbrock \& Ferland 2006) and equal velocity dispersions for the [N II] lines in our calculations. Finally, $\mathrm{Pa} \beta$ emission lines were fitted within the restframe range 12200-13200 $\mathrm{A}$, where we used a single Gaussian profile to describe the narrow component of $\mathrm{Pa} \beta$ emission line.

For fitting the BLR profiles, we used a single Gaussian model for some of AGN, but a second Gaussian profile was required to characterize the BLR profile for the following galaxies MCG05-14-12, MCG-06-30-15, NGC 3783, NGC 4593, NGC 4235, NGC 6814, and NGC 7213. For the broad-line profiles that required double Gaussian models, we combined both Gaussian profiles with each other, and the resulting full width at half maximum (FWHM) was estimated from the new, combined profile. Uncertainties of the FWHM estimates were derived from the fit residuals. We emphasize that the narrow emission line components and the AGN continuum were extracted before we estimated the width of broad emission line profiles. To test the reliability of the $\mathrm{H} \alpha$-based calculations, we additionally studied the $\mathrm{H} \beta$ and $\mathrm{Pa} \beta$ (when $\mathrm{H} \beta$ is not available) emission profiles for comparison. The resulting FWHM differences between the $\mathrm{H} \alpha$, $\mathrm{H} \beta$, and $\mathrm{Pa} \beta$ emission line profiles of our sample are found to be less than $20 \%$, and this result is consistent with other observational results from different sample (Greene \& Ho 2005; Shen \& Liu 2012; Mejía-Restrepo et al. 2016; Ricci et al. 2017b). For consistency, we used the same number of Gaussian models to fit $\mathrm{H} \alpha, \mathrm{H} \beta$, and $\mathrm{Pa} \beta$ emission line profiles of each AGN. The resulting parameters are presented in Table 3 .

In the case of MCG-05-14-12, NGC 1365 and NGC 2992 we detected blue-shifted emission lines in the spectra $\left(>500 \mathrm{~km} \mathrm{~s}^{-1}\right)$, which were also fitted with additional single Gaussian models. We excluded these blue-shifted emission lines, when we estimated our final BLR profiles of the LLAMA AGNs. We present the emission line fitting of our Type 1 AGN sample Fig. A.1.

MCG-05-14-12 and MCG-06-30-15 both show low emission line widths $\left(F W H M<1700 \mathrm{~km} \mathrm{~s}^{-1}\right)$ and low $[\mathrm{O}$ III] $] / \mathrm{H} \beta$ ratios (0.2 and 0.9 , respectively). According to the definition of narrow-line Seyfert 1 (NLS1) galaxies $\left(F W H M<2000 \mathrm{~km} \mathrm{~s}^{-1}\right.$ and $[\mathrm{O} \mathrm{III]} / \mathrm{H} \beta<3$ ) reported by Osterbrock \& Pogge (1985), we classify them as such.

\subsection{Black hole mass estimations}

By assuming virialized, rotating gas in the BLR that is gravitationally dominated, black hole masses can be obtained by

$M_{\mathrm{BH}}=f\left(\frac{\Delta V^{2} R}{G}\right)$,

where $f$ is a factor that depends on the unknown structure, kinematics, and orientation of the BLR, $\Delta V$ is the velocity dispersion of the broad emission line, $G$ is the gravitational constant, and $R$ is the BLR radius (e.g., Peterson et al. 2004). In this equation, the $f$ factor converts the observed virial product into black hole masses.

From the RM studies, a strong correlation between the AGN continuum luminosity $\left(\lambda L_{5100}\right)$ and the radius of the BLR $\left(R_{\mathrm{BLR}}\right)$ have been determined (Kaspi et al. 2000; Bentz et al. $2009 \mathrm{~b}, 2013)$. By adopting the $R_{\mathrm{BLR}}-\lambda L_{5100}$ relation, black hole masses based on virial single-epoch empirical correlations can be obtained. The tight empirical correlations between $M_{\mathrm{BH}}$ and emission from BLR regions can be expressed as

$$
\begin{aligned}
& M_{\mathrm{BH}}=10^{\alpha} \times\left(\frac{L_{\mathrm{H} \alpha}}{10^{42} \mathrm{erg} \mathrm{s}^{-1}}\right)^{\beta} \times\left(\frac{F W H M_{\mathrm{H} \alpha}}{10^{3} \mathrm{~km} \mathrm{~s}^{-1}}\right)^{\gamma} \times f_{F W H M} M_{\odot}, \\
& M_{\mathrm{BH}}=10^{\alpha} \times\left(\frac{\lambda L_{5100}}{10^{44} \mathrm{erg} \mathrm{s}^{-1}}\right)^{\beta} \times\left(\frac{\sigma_{\mathrm{H} \beta}}{10^{3} \mathrm{~km} \mathrm{~s}^{-1}}\right)^{\gamma} \times f_{\sigma} M_{\odot}, \\
& M_{\mathrm{BH}}=10^{\alpha} \times\left(\frac{L_{\mathrm{Pa} \beta}}{10^{42} \mathrm{erg} \mathrm{s}^{-1}}\right)^{\beta} \times\left(\frac{F W H M_{\mathrm{Pa} \beta}}{10^{4} \mathrm{~km} \mathrm{~s}^{-1}}\right)^{\gamma} \times\left(\frac{f_{\sigma}}{4.31}\right) M_{\odot},
\end{aligned}
$$

where we adopted the $\alpha, \beta, \gamma$ values $6.544,0.46,2.06$ for the $L_{\mathrm{H} \alpha}-F W H M_{\mathrm{H} \alpha}, 6.819,0.533,2.0$ for the $L_{5100}-\sigma_{\mathrm{H} \beta}$ calibration (Woo et al. 2015, hereafter W15), and 7.834, 0.46, 1.88 for the $L_{\mathrm{Pa} \beta}-F W H M_{\mathrm{Pa} \beta}$ calibration reported by La Franca et al. (2015). Since some studies suggest that the line profile of $\mathrm{H} \beta$ is not universal, and the second moment $\left(\sigma_{\text {Line }}\right)$ of $\mathrm{H} \beta$ profile gives more accurate $\mathrm{H} \beta$-based $M_{\mathrm{BH}}$ estimates (Peterson et al. 2004; 
Collin et al. 2006), we used $\sigma_{\text {Line }}$ for our $\mathrm{H} \beta$-based $M_{\mathrm{BH}}$ investigations. This effect is discussed in Sect. 4.3.

The observed flux of broad $\mathrm{H} \beta$ emissions weakens with the decrease of the inclination angle of AGN structure and becomes undetectable for Sy 1.9 galaxies (e.g., Schnorr-Müller et al. 2016). However, broad $\mathrm{H} \alpha$ can be observed even in these moderately obscured AGNs. Therefore, we estimate black hole masses of our sample using broad $\mathrm{H} \alpha$ emission lines for the entire sample, whereas we present the black hole masses obtained from $\mathrm{H} \beta$ or $\operatorname{Pa} \beta$ for comparison.

Furthermore, we adopted $M_{\mathrm{BH}}$ estimates of NGC 4388 and NGC 5728 obtained by Greene et al. (2016) and Braatz et al. (2015), respectively. Finally, the $M_{\mathrm{BH}}$ of NGC 5128 was adopted from Cappellari et al. (2009), in which the authors used stellar kinematics to obtain $M_{\mathrm{BH}}$ value. Therefore, we have $13 M_{\mathrm{BH}}$ estimates in total for ten Type 1 and three Type 2 AGNs, which will be further used in our $M_{\mathrm{BH}}$ and $\sigma_{\star}$ investigations.

\subsection{The $f$ factor}

We estimate the black hole masses for our sample using the broad-line-based single-epoch scaling relations. In the broadline-based black hole mass estimations, the dimensionless $f$ factor is an important parameter that can change the $M_{\mathrm{BH}}$ estimates by an order of magnitude. The obscurity of geometry, kinematics, and orientation of the BLR constitute systematic uncertainties encapsulated in the $f$ factor. Although there is no precise method to obtain the $f$ factor, it is determined in the literature by assuming AGN-hosting galaxies follow the inactive $M_{\mathrm{BH}}-\sigma_{\star}$ relation (e.g., Onken et al. 2004). A mean value of $f \sim 5$ is reported for $M_{\mathrm{BH}}$ estimations based on $\sigma_{\star}$ with an intrinsic scatter of $0.35 \mathrm{dex}$, whereas the $f$ factor is found to be $\sim 1$ for $M_{\mathrm{BH}}$ estimations based on FWHM (e.g., Woo et al. 2015; Grier et al. 2017).

Interestingly, Storchi-Bergmann et al. (2017) and MejíaRestrepo et al. (2018) show an anti-correlation between the $F W H M_{\text {obs }}$ and the $f$ factor, and Mejía-Restrepo et al. (2018) provide a relation for the $f$ factor calculations, i.e., $f=\left(F W H M_{\text {obs(line })} / F W H M_{\mathrm{obs}}^{0}\right)^{\beta}$, where $\beta$ and $F W H M_{\mathrm{obs}}^{0}$ values are $-1.0 \pm 0.10,4000 \pm 700 \mathrm{~km} \mathrm{~s}^{-1}$ for $\mathrm{H} \alpha$ and $-1.17 \pm 0.11$, $4550 \pm 1000 \mathrm{~km} \mathrm{~s}^{-1}$ for $\mathrm{H} \beta$, respectively. This formula is roughly consistent with the $f$ factor of 1.12 (W15), $f$ factor of 1.51 (Grier et al. 2013b) for both $\mathrm{H} \alpha$ and $\mathrm{H} \beta$ BLR gas with a FWHM in the range $2000-4000 \mathrm{~km} \mathrm{~s}^{-1}$, whereas the difference between calibrations significantly increases for the BLR gas with $F W H M<2000 \mathrm{~km} \mathrm{~s}^{-1}$. Accordingly, the $f$ factor is reported to be different for every AGN (Pancoast et al. 2014).

Until recently, there has been no direct method to obtain the $f$ factor, but interestingly the GRAVITY Collaboration (2018) resolves the BLR region of 3C 273 using observational data from VLTI/GRAVITY. In the same work, the authors report an $f_{F W H M}=1.3 \pm 0.2$ and $f_{\sigma}=4.7 \pm 1.4$ for $3 \mathrm{C} 273$. The GRAVITY Collaboration (2018) note that a comparison between $\mathrm{RM}$ and interferometry in the same objects can be very efficient for understanding the characteristics of BLRs and for increasing the accuracy of $M_{\mathrm{BH}}$ estimations. Even though the $f$ factor remains an uncertainty of $M_{\mathrm{BH}}$ estimations of Type 1 AGNs for now, the $f$ factor of $\sim 1$ and 5 are expected to represent the BLR structure for FWHM and $\sigma_{\text {Line }}$ estimations, respectively. Further investigations with VLTI/GRAVITY are required to resolve the BLR structures for each AGNs.

The latest single-epoch RM based calibrations are presented by Woo et al. (2015), and we use these for the further analysis: we adopt an $f$ factor of $4.47(\log f=0.65 \pm 0.12)$ for estimates based on $\sigma_{\text {Line }}$ of $\mathrm{H} \beta$ and $1.12(\log f=0.05 \pm 0.12)$ for estimates based on the modeled FWHM of $\mathrm{H} \alpha$, respectively. For the black hole mass estimates based on the Paschen- $\beta$ line, we recalibrate the La Franca et al. (2015) calibration adopting the same $f$ factor as for the $\mathrm{H} \beta$ estimate.

\subsection{Dust extinction}

In the single-epoch RM calibration, the luminosity is usually not corrected for extinction since the objects studied there are essentially unobscured (Type 1) AGNs. Since we also have moderately obscured Type 1 objects in our sample, an extinction correction must be applied to these objects to have accurate $M_{\mathrm{BH}}$ estimations. In a previous LLAMA project, Schnorr-Müller et al. (2016) use the line ratios of various hydrogen recombination lines from the UV to the NIR to derive both the excitation conditions and the optical extinction to the BLR for nine objects. We adopt $A_{V}$ (BLR) estimates from Schnorr-Müller et al. (2016) for nine of the Type 1 AGNs in our sample. We note that $A_{V}(\mathrm{BLR})$ of NGC 7213 is obtained in this study using the same approach provided by Schnorr-Müller et al. (2016). This method can only be used for Type 1 AGNs, and a more detailed explanation for extinction calculation is given by Schnorr-Müller et al. (2016).

It is worth mentioning that Burtscher et al. (2016) and Shimizu et al. (2018) also estimate the extinction in the BLR by comparing X-ray absorption and optical obscuration for some AGNs in our sample. The estimated $A_{V}(\mathrm{BLR}) \mathrm{s}$ are found to be consistent with those reported by Schnorr-Müller et al. (2016). Since the method from Schnorr-Müller et al. (2016) is a more direct method for obtaining the BLR extinction, we used their $A_{V}$ (BLR) estimates.

In order to convert from $A_{V}$ to the extinction at a any wavelength $\left(A_{\lambda}\right)$, we employ the extinction law presented by Wild et al. (2011) as follows:

$A_{\lambda} / A_{V}=0.6(\lambda / 5500)^{-1.3}+0.4(\lambda / 5500)^{-0.7}$

In this equation, the first term describes the dust extinction along the line of sight (assuming Milky Way dust), whereas the second term provides the dust extinction caused by the diffuse interstellar medium. Wild et al. (2011) reports that this equation provides a good correction for AGNs with a large dust reservoir. We used Eq. (6) to convert the BLR extinction in $V$ band to the BLR extinction in $\mathrm{H} \alpha(6562.8 \AA), \mathrm{H} \beta(4861.4 \AA)$ and $\mathrm{Pa} \beta$ (1281.8 $\AA$ ). As mentioned in Schnorr-Müller et al. (2016), this relation gives a good correction for both the NLR and BLR of the LLAMA AGNs.

The resulting $A_{\lambda}(\mathrm{BLR})$ values are used to correct the extinguished BLR flux (S) of $\mathrm{H} \alpha$ and the continuum flux of $L_{5100}$ using the following equation:

$S_{\text {corrected }}=S_{\text {observed }} \times 10^{0.4 A_{\lambda}}$.

For highly obscured sources in our sample (NGC 1365, NGC 2992, MCG-05-23-16), we used $\mathrm{Pa} \beta M_{\mathrm{BH}}$ calibration reported by La Franca et al. (2015) (see Eq. (4)) for obtaining $M_{\mathrm{BH}}$ values, since the broad $\mathrm{H} \beta$ cannot be detected for these sources. Even though the NIR band suffers less from the dust extinction (Landt et al. 2013), we also corrected the slightly extinguished BLR flux of $\mathrm{Pa} \beta$ using the resulting $A_{\lambda}(\mathrm{BLR})$ in our calculations. 


\subsection{Accretion rate}

In this section, we explain the method for estimating the Eddington ratios and accretion rates of our sample by adopting the following empirical relations. First, we obtain the bolometric luminosities by Winter et al. (2012), i.e.,

$\log L_{\mathrm{Bol}}=1.12 \log L_{14-195 \mathrm{keV}}-4.23 \mathrm{erg} \mathrm{s}^{-1}$.

Then, the Eddington luminosity $\left(L_{\mathrm{Edd}}\right)$ can be written as $L_{\text {Edd }}=1.26 \times 10^{38} M_{\mathrm{BH}} / M_{\odot}$ (Rybicki \& Lightman 1986). We used our single-epoch $M_{\mathrm{BH}}$ values from $\mathrm{H} \alpha$ to estimate the Eddington luminosities for the Type 1 sources. To obtain Eddington luminosities for the LLAMA Type 2 sources, we used black hole masses that are calculated from the LLAMA $M_{\mathrm{BH}}-\sigma_{\star}$ relation (see Sect. 4.6), whereas we collected the megamaser black hole masses for NGC 4388 Greene et al. (2016) and NGC 5728 (Braatz et al. 2015), respectively. The Eddington ratio $\left(\lambda_{\text {Edd }}\right)$ can be computed by

$\lambda_{\mathrm{Edd}}=\left(\frac{L_{\mathrm{Bol}}}{L_{\mathrm{Edd}}}\right)$.

Finally, the mass accretion rate $(\dot{M})$ onto the black hole can be estimated by assuming a steady radiative efficiency $\epsilon=0.1$ (Collin \& Huré 2001), i.e.,

$\dot{M}=\left(\frac{L_{\mathrm{Bol}}}{\epsilon c^{2}}\right)$.

We note that the main contribution to uncertainty on the Eddington ratios and accretion rates originate from the uncertainty in bolometric luminosity, accretion efficiency and $M_{\mathrm{BH}}$, which corresponds to an uncertainty of $\sim 0.4-0.5$ dex (Bian \& Zhao 2003; Marinucci et al. 2012). This uncertainty range is roughly consistent with the median value of our estimates. The resulting Eddington and mass accretion rates can be found in Table 3.

\subsection{Statistical fitting procedure}

The FITEXY, an IDL-based tool, developed by Press et al. (1992) and modified by Tremaine et al. (2002), is an effective tool for estimating fit parameters for a linear regression model. The original idea of the FITEXY method is based on a modified version of bivariate correlated errors and intrinsic scatter proposed by Akritas \& Bershady (1996). The FITEXY method minimizes the $\chi^{2}$ statistic and takes into account the measurement error for both dependent or independent variables for $\mathrm{X}$ and $\mathrm{Y}$ axes. In this method, $\chi^{2}$ is minimized by

$\chi^{2}=\sum_{i=1}^{N} \frac{\left(\mu_{i}-\alpha-\beta s_{i}\right)^{2}}{\sigma_{\mu, i}^{2}+\beta \sigma_{s, i}^{2}+\epsilon_{0}^{2}}$,

where $\mu$ is $\log \left(M_{\mathrm{BH}} / M_{\odot}\right), s$ is $\log \left(\sigma_{\star} / \sigma_{0}\right)$, where $\sigma_{0}$ is $200 \mathrm{~km} \mathrm{~s}^{-1}, \sigma_{\mu}$ and $\sigma_{s}$ are measurement uncertainties in both variables, and $\epsilon_{0}$ is the intrinsic scatter.

To fit the $M_{\mathrm{BH}}-\sigma \star$ relation, we used a single power law as expressed in the following equation:

$\log \left(M_{\mathrm{BH}} / M_{\odot}\right)=\alpha+\beta \log \left(\frac{\sigma_{\star}}{\sigma_{0}}\right)$,

where $\alpha$ is the intercept, $\beta$ is the slope of the single power-law fit. We emphasize that both $M_{\mathrm{BH}}$ and $\sigma_{\star}$ parameters are estimated using the data obtained from the same spectra for the LLAMA Type 1 sources.

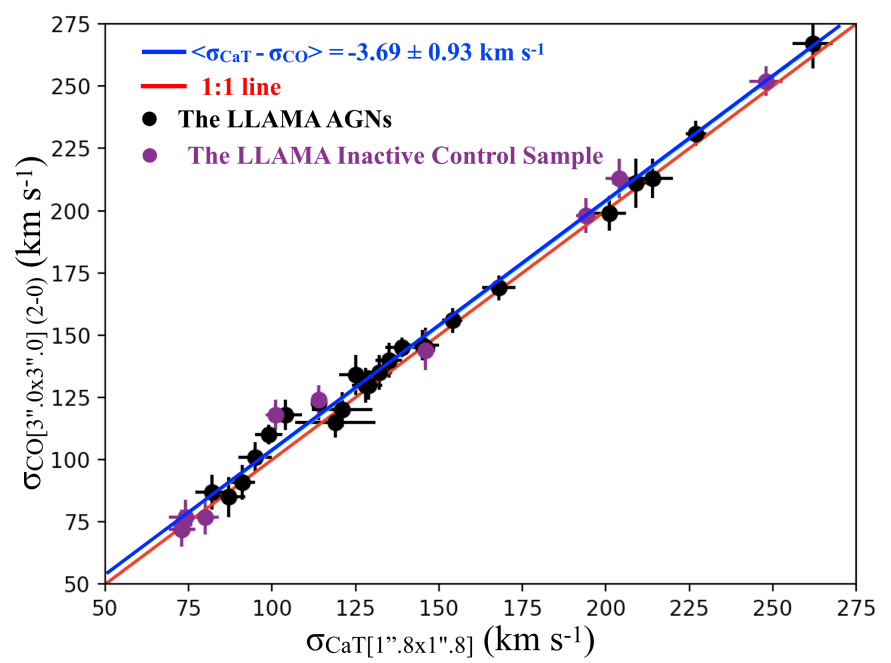

Fig. 1. Stellar velocity dispersion results, which are calculated from the $\mathrm{CaT}$ and CO (2-0) absorption features. Some of sources are not still observed for our entire sample, therefore, the sources for which our sample includes both $\sigma_{\mathrm{CaT}}$ and $\sigma_{\mathrm{CO}(2-0)}$ estimates are compared. The red solid line represents 1:1 line, whereas the blue solid line shows the offset between the $\sigma_{\mathrm{CaT}}$ and $\sigma_{\mathrm{CO}(2-0)}$ estimates of our data. The LLAMA AGNs and inactive galaxies are presented as black and purple, respectively.

\section{Results and discussion}

In this section we first study and discuss the robustness of the observables and assumptions involved in constructing the $M_{\mathrm{BH}^{-}}$ $\sigma_{\star}$ relation, before presenting the $M_{\mathrm{BH}}-\sigma_{\star}$ relation for our sample.

\subsection{Stellar velocity dispersion estimates: Optical versus near-infrared}

We provide stellar velocity dispersion estimates of the CaT absorption lines; these results are in the range $73 \leq \sigma_{\star \text { CaT }} \leq$ $227 \mathrm{~km} \mathrm{~s}^{-1}$ for our sample of AGNs (see Table 2). Besides, the estimated $\sigma_{\star \text { CaT }}$ values for the LLAMA inactive sample are found to be $64 \leq \sigma_{\star \mathrm{CaT}} \leq 262 \mathrm{~km} \mathrm{~s}^{-1}$. This shows that the LLAMA active and inactive subsamples, which are matched on total stellar mass ( $H$ band luminosity), also have comparable bulge stellar masses.

Alternatively, we estimated the stellar velocity dispersion from the NIR CO (2-0) absorption band head using the SINFONI data for a comparison. The $\sigma_{\star} \mathrm{CO}(2-0)$ values are found to be slightly higher $\left(\sim 3.69 \pm 0.93 \mathrm{~km} \mathrm{~s}^{-1}\right)$ than the $\sigma_{\star \text { CaT }}$. The most likely explanation for this is that the NIR CO feature probes more deeply embedded (and therefore higher velocity dispersion) stellar populations than the optical CaT. Our result shows a different trend than the result from Riffel et al. (2015), who claim that the discrepancy between $\sigma_{\star} \mathrm{CO}(2-0)$ and $\sigma_{\star \mathrm{CaT}}$ is higher $\left(\left\langle\sigma_{\star \mathrm{CO}(2-0)}\right\rangle-\left\langle\sigma_{\star \mathrm{CaT}}\right\rangle=19 \pm 6 \mathrm{~km} \mathrm{~s}^{-1}\right)$. In previous works, $\sigma_{\star \mathrm{CaT}}$ estimates are found to be equal to $\sigma_{\star} \mathrm{CO}(2-0)$ estimates for earlytype galaxies (e.g., Silge \& Gebhardt 2003; Rothberg \& Fischer 2010). Interestingly, these results are consistent with our result for late-type dominated LLAMA sample. The $\sigma_{\star} \mathrm{CO}(2-0)$ versus $\sigma_{\star \text { CaT }}$ comparison and the resulting parameters are presented in Fig. 1 and Table 2.

\subsection{Robustness of stellar velocity dispersion estimations}

Recent studies report that stellar velocity dispersion estimates can be affected by AGN contamination (Greene \& Ho 2006b; 
Harris et al. 2012; Woo et al. 2013; Batiste et al. 2017b). Firstly, we address the question of whether the AGN continuum affects the stellar velocity dispersion estimations. In optical bands, the AGN continuum behaves like a power-law function (Oke et al. 1984) and can be defined as $f_{\lambda} \propto \lambda^{-\left(\alpha_{v}+2\right)}$, where $\alpha_{v}$ is the arithmetic mean of the power-law index. We adopt $\alpha_{v}=-2.45$ (Vanden Berk et al. 2001) to model a synthetic AGN continuum. First, we selected an inactive control galaxy (NGC 1315) from the LLAMA sample; the stellar velocity dispersion of this galaxy is estimated as $\sigma_{\star}=77 \pm 5 \mathrm{~km} \mathrm{~s}^{-1}$ using pPXF. Then, the synthetic AGN continuum was combined with the NGC 1315 spectrum. As expected, the AGN continuum has no direct effect on the $\sigma \star$ estimations for any reasonable AGN continuum level $(<70 \%)$, if the continuum is modeled using an adequate number of additive polynomials. In the top panel of Fig. 2, we present a synthetic AGN spectrum, which consists of the spectrum of the inactive galaxy NGC 1315 (shown as red line) and a fairly strong ( 70\%) model AGN continuum (blue line). Our active galaxies typically show a much smaller AGN contribution than $70 \%$ at the CaT, which is why this serves as a good test for our fitting accuracy.

On the other hand, the continuum level cannot be estimated accurately, if the spectrum is noisy. To test this, we applied a Monte Carlo approach to generate noise for every pixel of the synthetic AGN spectra. In this approach, a normal distribution of numbers are allowed to vary within a specified range, and the test was repeated $10^{4}$ times to obtain the mean distribution of each noise level (S/N: 3, 5, 10, 15, 25, 50, 100). For each S/N level, we fit the data $10^{2}$ times using pPXF. The stellar velocity dispersion estimates are obtained from the mean of the Gaussian distribution of resulting $\sigma_{\star}$ values for each $\mathrm{S} / \mathrm{N}$. In Fig. 2 (middle), we present the comparison between $\mathrm{S} / \mathrm{N}$ and $\sigma_{\star}$ estimates. By considering this result, we can achieve reliable $\sigma_{\star}$ estimations using data with high $\mathrm{S} / \mathrm{N}(>15)$. We confirm that $\mathrm{S} / \mathrm{N}$ is one of the most important factors, leading to an uncertainty of up to $20 \%$ for a $S / N \lesssim 5$, which needs to be included into the total uncertainty of $\sigma_{\star}$. We note that our sample of AGNs are observed with $S / N>40$; therefore, our calculations are not affected by this issue.

Moreover, AGN emission lines can also affect $\sigma_{\star}$ estimations. The broad O I $(8446 \AA)$ emission line, which is detected for some of the AGNs in our sample, is a good example of this (see the bottom Fig. 2). Correspondingly, we modeled an extremely broad O I $8446 \AA$ line using a Gaussian model $\left(\sigma_{\text {OI }} \sim 2500 \mathrm{~km} \mathrm{~s}^{-1}\right)$, which we added to the synthetic AGN spectrum. By fitting spectra around the $\mathrm{CaT}$ regime with different noise levels, we find evidence that the broad O I $8446 \AA$ emission line can cause inaccurate stellar velocity dispersion estimations of up to $15 \%$. Since the existence of a broad emission line affects the continuum level determination, such AGNs with broad O I $8446 \AA$ have been treated specially by masking the part of the spectrum that is affected by the emission line. In a few cases, this can cut off the first CaT line ( $8498 \AA$ ), but we report that this does not affect the determination of the stellar velocity dispersion.

For disk galaxies, the galaxy rotation makes an important contribution to the measured stellar velocity dispersion from a larger aperture. The rotational dynamics of spiral galaxies are characterized by the total luminosity, line of sight, and maximum rotation velocities of the galaxy and the inclination angle of the disk (Tully \& Fisher 1977). Since the LLAMA AGN sample is dominated by spiral galaxies, the galaxy rotation is another effect that may affect the stellar velocity dispersion estimates. By using the velocity-shifted SINFONI data cubes from Shimizu et al. (in prep.), we obtained an average inclination-corrected rotational velocity for the LLAMA sample.The contribution from the rotational effects are further discussed in Sect. 4.7.
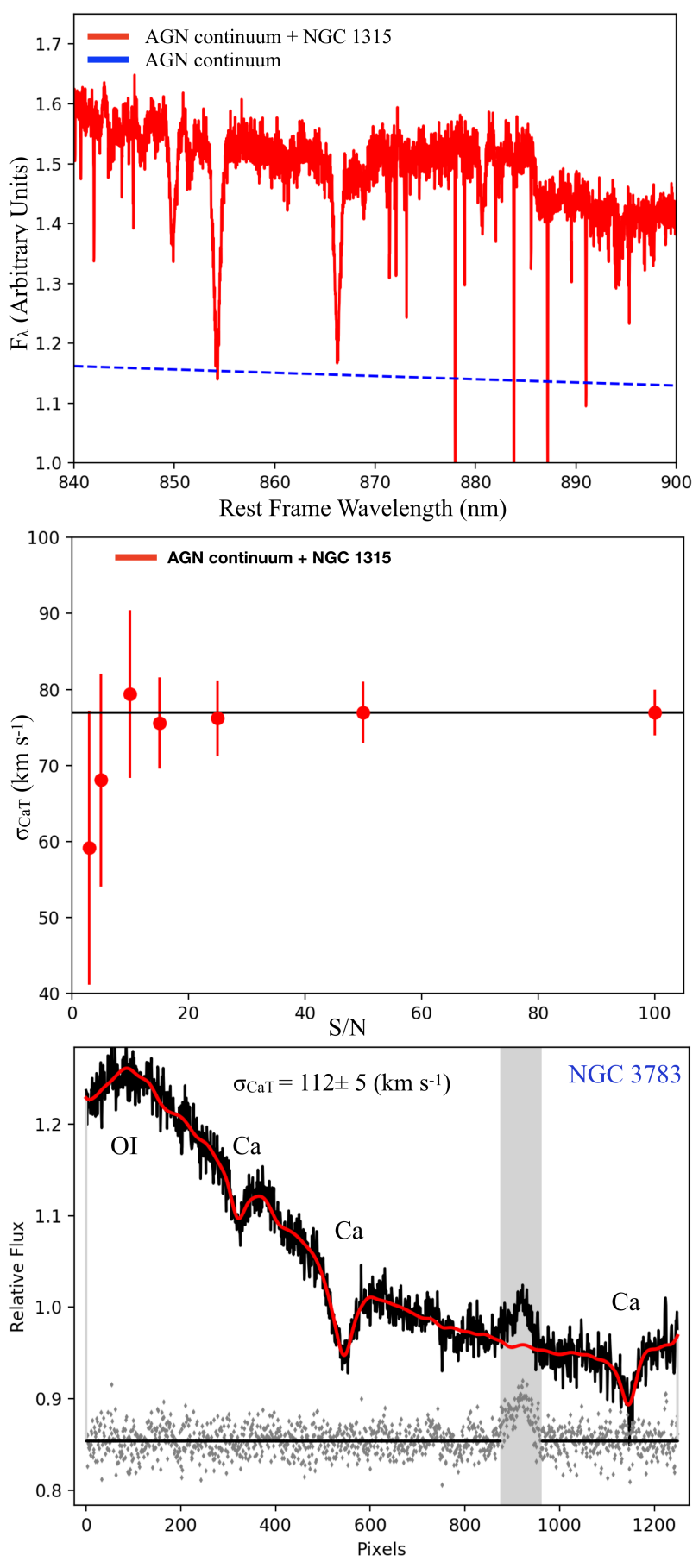

Fig. 2. Top: example of the spectrum from the control galaxy NGC 1315, which is combined with the model AGN continuum. The assumed AGN continuum are presented as the red and the blue, respectively. Middle: stellar velocity dispersion estimates relative to the $\mathrm{S} / \mathrm{N}$ of the AGN continuum for NGC 1315 (red). The solid black line represents the stellar velocity dispersion estimate from the X-shooter spectrum, which has a $S / N \sim 44$ per pixel. Bottom: example of pPXF fit for NGC 3783. Position of the O I emission line and the CaT absorption lines are demonstrated in the plot for visual aid. The gray masked feature represents the Fe II emission line at $8616 \AA$.

\subsection{Robustness of broad-line-based $M_{\mathrm{BH}}$ estimates}

We investigated the broad-line emission of our sample of Type 1 AGNs using two different apertures: $0 \prime \prime 6 \times 0$ "' 6 (the central region) and 1 ". $8 \times 4^{\prime \prime}$ (the FOV of X-shooter data). For each $\mathrm{AGN}$, we fit $\mathrm{H} \alpha$ and $\mathrm{H} \beta$ emission lines with the same number 


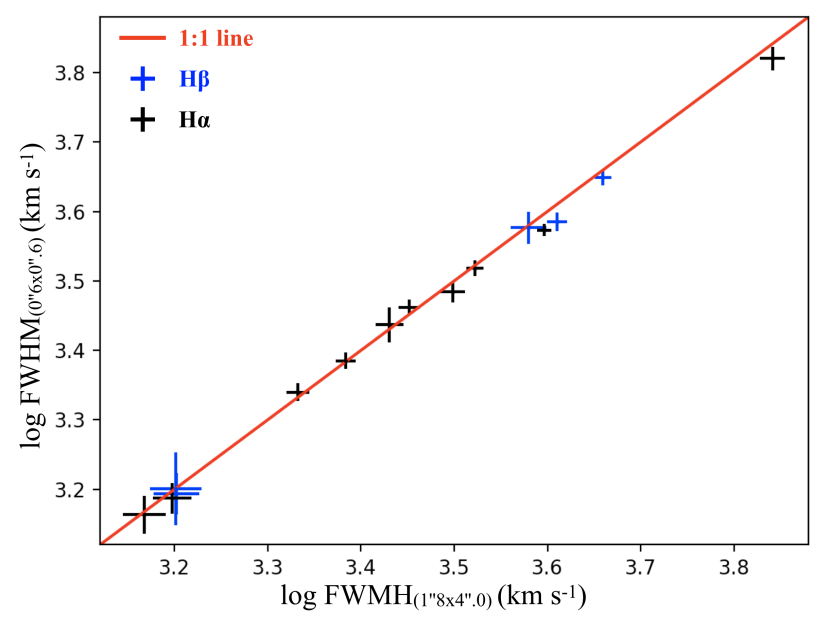

Fig. 3. Resulting FWHM comparisons for the small $\left(00^{\prime \prime} 6 \times 00^{\prime \prime} 6\right)$ and big aperture $\left(1 " .8 \times 4^{\prime \prime}\right)$ for our sample. The black marker represents the resulting estimates from the $\mathrm{H} \alpha$, whereas the blue marker indicates obtained results from $\mathrm{H} \beta$. The black solid line indicates the 1:1 line.

of the Gaussian curves for each aperture. In Fig. 3, we present FWHM comparisons between the central region and the FOV. The broad-line FWHM estimates are found to differ up to $5 \%$ as a consequence of aperture choice. This difference can be related to the observational seeing or the narrow-line contamination. Since we cannot detect the entire BLR gas, this is a systematic error of FWHM estimates and should be added to total uncertainty budget of FWHM estimates.

The emission line width of a broad line can be obtained either from the FWHM or line dispersion $\left(\sigma_{\text {Line }}\right)$. A typical AGN emission line profile can be described by a single Gaussian profile, and $F W H M / \sigma_{\text {Line }}$ has a fixed ratio of $2 \sqrt{2 \ln 2} \approx 2.355$ in the Gaussian profile. However, some of AGN emission line widths can only be modeled with multiple Gaussians. In this case, the FWHM needs to be estimated from the combined Gaussian models, and the ratio of FWHM to $\sigma_{\text {Line }}$ can vary (Peterson et al. 2004; Peterson 2011). Peterson \& Bontà (2018) argue that estimations based on $\sigma_{\text {Line }} M_{\mathrm{BH}}$ are more accurate than those based on FWHM for $\mathrm{H} \beta$, if an AGN emission has an irregular line profile. For the multiple-peaked emission line profiles, the irregular kurtosis can be either positive or negative, and it can affect the accuracy of emission line estimations. These authors also note that estimations based on $\sigma_{\text {Line }}$ are less sensitive to the contribution from extended line wings. The $\sigma_{\text {Line }}$ can be estimated from the second moment of the emission line profile $P(\lambda)$ as follows:

$\sigma_{\text {Line }}=\left[\int \frac{\left(\lambda-\lambda_{0}\right)^{2} P(\lambda) \mathrm{d} \lambda}{\int P(\lambda) \mathrm{d} \lambda}\right]^{1 / 2}$,

where $\lambda_{0}$ is the center of emission line profile. In Fig. 4, we compare the $\sigma_{\text {Line }}$ obtained from the Eq. (13) and $\sigma_{\text {Model }}$ obtained from its ratio with the FWHM $\left(F W H M / \sigma_{\text {Model }} \approx 2.355\right)$ for the Gaussian profile. We find a slight difference (an offset of $76.7 \pm 56.2 \mathrm{~km} \mathrm{~s}^{-1}$ ) between the two estimates for our $\mathrm{H} \beta$-based investigations. We note that this difference affects our $M_{\mathrm{BH}}$ estimates by $\sim 0.1$ dex. This result is consistent with Peterson \& Bontà (2018), therefore, we also suggest using $\sigma_{\text {Line }}$ in investigations based on $\mathrm{H} \beta M_{\mathrm{BH}}$.

\subsection{Black hole masses and the systematical uncertainties}

The $M_{\mathrm{BH}}$ values for our sample of Type 1 AGNs are presented in Table 3. They are in the range $6.34 \leq \log M_{\mathrm{BH}} \leq 7.75 M_{\odot}$ for

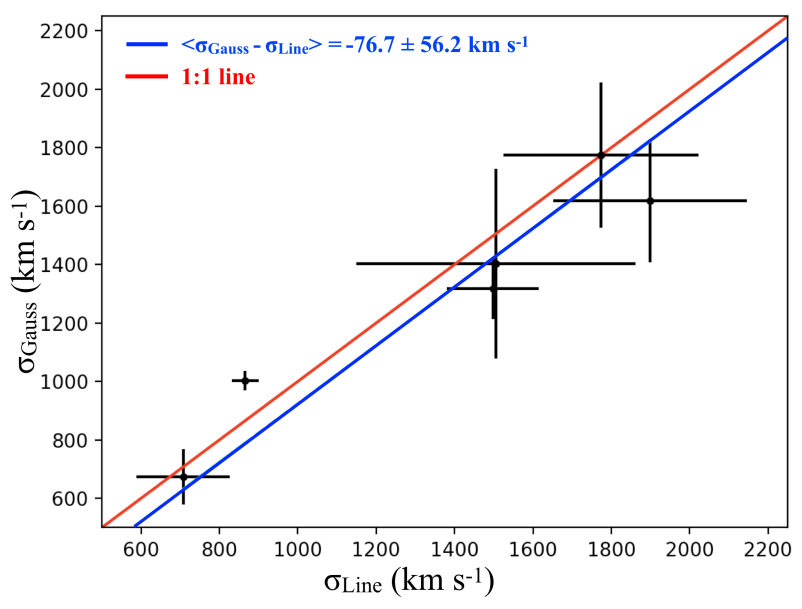

Fig. 4. Comparison between $\sigma_{\text {Line }}$, which is obtained from the Eq. (13) and $\sigma_{\text {Gauss }}$, which is obtained from the line width of Gaussian model.

$\mathrm{H} \alpha$. We note that the average black hole mass of inactive galaxies in the relation by Kormendy \& Ho (2013) is substantially higher, possibly indicating that our sample of AGNs did not yet go through a major merger phase (Wandel et al. 1999).

Black hole mass uncertainties are determined from the bootstrapping analysis. In this approach, we used all uncertainties from the parameters, such as uncertainties from single-epoch calibration parameters, $f$ factor, FWHM, and luminosity. First, we generated $10^{8}$ random numbers from a normal distribution for each parameter. Then, these numbers were added to all parameters of $M_{\mathrm{BH}}$ estimations. Finally, using the Gaussian distribution of obtained $10^{8} M_{\mathrm{BH}}$ values, we measured black hole mass uncertainties within the $1 \sigma$ confidence level.

However. single-epoch based $M_{\mathrm{BH}}$ estimations have been reported to have a systematical uncertainty, which is reported as a lower limit of 0.40 dex by (e.g., Pancoast et al. 2014). The uncertainty of $f$ factor introduces an uncertainty of 0.12 dex (Woo et al. 2015), which is obtained from the comparison of the $M_{\mathrm{BH}}-\sigma_{\star}$ relation between the RM AGNs and inactive galaxies. The second uncertainty is the intrinsic scatter of BLR radiusluminosity relation, which is reported as 0.13 dex for reliable estimates (Bentz et al. 2013). Third, the variability in luminosity and line width bring a 0.1 dex uncertainty (Park et al. 2012 b). Last, we adopt an uncertainty of 0.15 dex, which is assumed to come from redshift-independent distance measurements. Correspondingly, the total uncertainty of $M_{\mathrm{BH}}$ estimates can be $0.3-0.4$ dex.

\subsection{Accretion rates}

Many properties of the AGN (e.g., the torus phenomenology, Wada 2012) are expected to depend on the Eddington ratio of the "central engine". One of the main drivers of our study is to provide the Eddington ratio for the whole LLAMA sample.

We compute the accretion rates following Eqs. (8) and (10) and find them in the range $0.04<\dot{M}<0.92 M_{\odot} \mathrm{yr}^{-1}$ assuming an accretion efficiency of $10 \%$ (see Table 3). Using our estimated black hole masses, we further calculate the Eddington ratio $\lambda$ following Eq. (9) for all of our AGNs. They are in the regime $0.004 \leq \lambda \leq 0.49$. These results indicate that the most LLAMA AGNs are growing at a rate that is well below Eddington, although likely in the radiatively efficient regime via a geometrically thin, optically thick disk (Shakura \& Sunyaev 1973). 

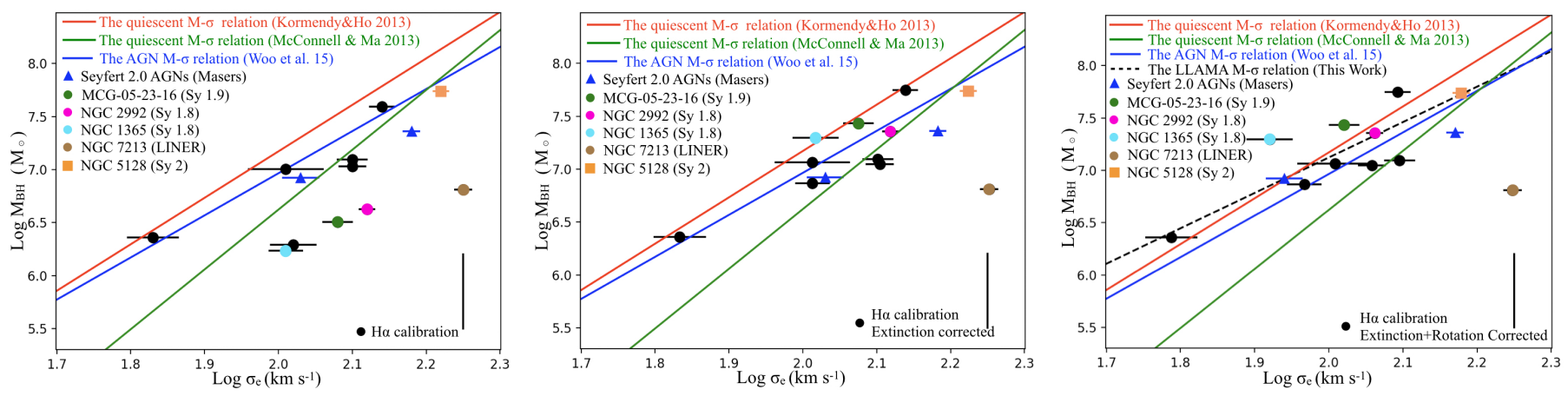

Fig. 5. Left: $M_{\mathrm{BH}}-\sigma_{\star}$ relation of our sample of galaxies, where $M_{\mathrm{BH}}$ values are estimated using the $\mathrm{H} \alpha$-based calibration. The $M_{\mathrm{BH}}-\sigma_{\star}$ relation of KH13, MM13, and W15 are presented as red, green, and blue solid lines, respectively. Sy 1.8, Sy 1.9, Sy 2, and LINER galaxies are presented in different colors for visual aid. The location of the two LLAMA Seyfert 2 (NGC 4388 and NGC 5728) galaxies are shown that have megamaser $M_{\mathrm{BH}}$ estimates as blue triangles. In addition, we present the $M_{\mathrm{BH}}$ estimates of NGC 5128 obtained from stellar kinematics as an orange box (Cappellari et al. 2009). Finally, the average uncertainties on the black hole mass estimates of the LLAMA AGNs ( $\sim .40$ dex) are presented as a vertical black line in the legend to avoid confusion of data points. Middle: $M_{\mathrm{BH}}-\sigma_{\star}$ relation of our sample of galaxies, where $M_{\mathrm{BH}}$ values are estimated using the extinction-corrected fluxes and the $\mathrm{H} \alpha$ calibration. Right: $M_{\mathrm{BH}}-\sigma_{\star}$ relation of our sample of galaxies, where the $\mathrm{H} \alpha M_{\mathrm{BH}}$ values are presented as the extinction-corrected and $\sigma_{\star}$ values are presented as rotation-corrected. The LLAMA $M_{\mathrm{BH}}-\sigma_{\star}$ relation is presented as a black dashed line.

\subsection{LLAMA sample $M_{\mathrm{BH}}-\sigma_{\star}$ relation}

In Fig. 5, we present the $M_{\mathrm{BH}}-\sigma_{\star}$ relation for the LLAMA AGN sample adopting the broad-line-based single-epoch black hole masses derived using the $\mathrm{H} \alpha$ emission line profiles. Using the high $\mathrm{S} / \mathrm{N}$ data, we report 38 stellar velocity dispersion estimates (20 AGNs and 18 inactive galaxies) in total (Table 2), which are derived from the CaT and/or CO (2-0) absorption features. We provide $M_{\mathrm{BH}}$ of 10 Type 1 AGNs in the LLAMA sample (see Table 3). In addition, we adopt a stellar kinematic $M_{\mathrm{BH}}$ estimate of NGC 5128 (Cappellari et al. 2009) and two megamaser $M_{\mathrm{BH}}$ estimates of NGC 4388 (Greene et al. 2016) and NGC 5728 (Braatz et al. 2015). Therefore, we constructed an $M_{\mathrm{BH}}-\sigma_{\star}$ relation for 13 AGNs in the LLAMA sample.

We then performed a linear regression in which we allowed both the intercept and the slope to vary. For this fit, we used FITEXY and the extinction-corrected $M_{\mathrm{BH}}$ and the rotationcorrected $\sigma_{\star}$ estimates for our sample. We excluded NGC 7213 from this fit since it shows LINER-like properties; also the $\mathrm{H} \beta$ fit for this galaxy fails.

The resulting $M_{\mathrm{BH}}-\sigma_{\star}$ relation for the LLAMA AGNs is written as

$\log \left(M_{\mathrm{BH}} / M_{\odot}\right)=8.14( \pm 0.20)+3.38( \pm 0.65) \log \left(\frac{\sigma_{\star}}{200 \mathrm{~km} \mathrm{~s}^{-1}}\right)$

and the intrinsic scatter of this relation is $\epsilon=0.32 \pm 0.06$. We note that our slope $(3.38 \pm 0.65)$ is smaller than the slope reported by Woo et al. (2015) (3.97 \pm 0.56$)$ who included narrow-line Seyfert AGNs in order to extend to lower black hole masses; our slope is consistent with Woo et al. (2013) who found a slope of $3.46 \pm 0.61$. Within the uncertainties of our small sample, our slope is consistent with both of these AGN relations, but not consistent with the slope reported by Kormendy \& Ho (2013) for more massive, inactive galaxies. This result still shows that the LLAMA sample of AGNs, which is a volume complete sample of the most luminous local AGNs, is representative for the larger AGN population sampled with RM in terms of its location and slope on the M-sigma relation.

For reference for future publication, and using the LLAMA $M_{\mathrm{BH}}-\sigma_{\star}$ relation (Eq. (14)), we also estimate $M_{\mathrm{BH}}$ values for our Type 2 AGNs (Table 3 ).

\subsection{LLAMA $M_{\mathrm{BH}}-\sigma_{\star}$ relation versus spheroidal $M_{\mathrm{BH}}-\sigma_{\star}$ relation}

In the left panel of Fig. 5, we present the $M_{\mathrm{BH}}$ values without extinction correction and $\sigma_{\star}$ parameter without rotation correction. We compare the LLAMA $M_{\mathrm{BH}}-\sigma_{\star}$ relation with the $M_{\mathrm{BH}^{-}}$ $\sigma_{\star}$ relation of KH13, MM13 and the AGN $M_{\mathrm{BH}}-\sigma_{\star}$ relation by W15. First, we found a high offset $(0.75$ dex $)$ from the KH13 relation using these parameters.

In previous works, some authors concentrated on correcting the broad Balmer fluxes and/or the monochromatic accretion luminosities in various wavelengths (i.e., 1350, 3000, $5100 \AA$ ), which are used in single-epoch $M_{\mathrm{BH}}$ estimations, using Galactic extinction maps (e.g., Vestergaard \& Peterson 2006; Denney et al. 2009; Shen \& Liu 2012; Bentz et al. 2016; Kozłowski 2017). In our study, we additionally corrected $\mathrm{H} \alpha$ and the continuum fluxes, which are used for deriving black hole masses, using the estimated BLR extinction of LLAMA sample by Schnorr-Müller et al. (2016). In the middle panel of Fig. 5, we present the $M_{\mathrm{BH}}-\sigma_{\star}$ relation obtained using extinction-corrected black hole masses. The extinction correction increased the estimated $M_{\mathrm{BH}}$ by a factor of $0.02-0.93$ dex for our sample, and reduced the average offset from the KH13 relation to 0.38 dex. This result indicates that the extinction in BLR can cause significantly under-estimation of $M_{\mathrm{BH}}$, unless it is taken into account.

In an upcoming LLAMA study, Shimizu et al. (in prep.) fit for the spatially resolved stellar kinematics within the SINFONI cubes. The stellar velocity fields are then modeled as an exponential disk. Using the model velocity field, we then shifted the spectra within the original SINFONI cubes such that the stellar velocity is removed. In this way, we can measure a rotation-corrected stellar velocity dispersion for the whole SINFONI FOV and compare this to the original value to produce a rotation correction that can be applied to our velocity dispersion based on X-shooter. Correspondingly, we obtain a rotation-correction factor for our AGNs (see Table 2). Therefore, we reduced the obtained stellar velocity dispersion using this rotation-correction factor. However, We are still missing SINFONI observations for the following galaxies: MCG-0514-12, NGC 4235, NGC 5128, and the spatially resolved stellar kinematics for NGC 3783, MCG-06-30-15. For these galaxies, the obtained stellar velocity dispersion estimates are reduced $10 \%$ the average galaxy rotation contribution to $\sigma \star$ for the 
LLAMA sample (Shimizu et al. in prep.). After the $\sigma_{\star}$ estimates are corrected for galaxy rotation, the LLAMA galaxies are found to agree with the $M_{\mathrm{BH}}-\sigma_{\star}$ relation of Kormendy \& Ho (2013). The average intrinsic scatter of LLAMA sample obtained adopting the slope and intercept of Kormendy \& Ho (2013) relation is found to be is $0.30 \mathrm{dex}$, which is consistent with the intrinsic scatter of the Kormendy \& Ho (2013) $M_{\mathrm{BH}^{-}} \sigma_{\star}$ relation (see Fig. 5). This result shows that the rotation can make a significant contribution to stellar velocity dispersion (up to 20\%), which is consistent with previous investigations (e.g., Kang et al. 2013; Batiste et al. 2017a; Eun et al. 2017).

We additionally compared our results with the $M_{\mathrm{BH}}-\sigma \star$ relation reported by MM13. By adopting a slope of 5.64 reported by MM13, we find an average offset of 0.46 dex for our sample relative to the relation of MM13. However, the majority of our sample ( 8 out of 10 ) are found to be above the relation reported by MM13. There are two possible explanations for the discrepancy between our results and MM13; in the MM13 sample, the disk galaxies are not corrected for their rotation component, and their sample includes brightest cluster galaxies, which are located in a different environment than the LLAMA sample.

Even though a few studies in the literature report that pseudobulges do not follow the $M_{\mathrm{BH}}-\sigma \star \sigma_{\star}$ relation (Greene et al. 2010; Kormendy et al. 2011; Kormendy \& Ho 2013), the pseudobulge-dominated LLAMA sample follows the $M_{\mathrm{BH}}-\sigma_{\star}$ relation of elliptical and spheroidal bulge-dominated galaxies after applying the extinction correction to our $M_{\mathrm{BH}}$ and the rotation correction to our $\sigma_{\star}$ estimates. Therefore, we argue that to reduce the offset from the elliptical-dominated $M_{\mathrm{BH}}-\sigma_{\star}$ relation, a correction to $M_{\mathrm{BH}}$ for the dust extinction (derived via the $\mathrm{H} \alpha$ or continuum flux) and a correction of $\sigma_{\star}$ for a rotational component of the disk/bulge must be applied to spiral-dominated local Seyfert AGNs.

\section{Conclusions}

In a volume-limited complete sample of the most luminous, X-ray selected, local Sy 1 AGNs, comprising the LLAMA sample, we examine the spatially resolved stellar kinematics and the properties of the broad emission lines using medium spectral resolution $(R \sim 8000) \mathrm{X}$-shooter data. We additionally compare our results with SINFONI data, which extend our analysis to the $\mathrm{H}+\mathrm{K}$ bands. Our main results are as follows:

- We obtain the stellar velocity dispersions via the CaT at $\sim 8500 \AA$; these are in the range $73 \leq \sigma_{\star \mathrm{CaT}} \leq 227 \mathrm{~km} \mathrm{~s}^{-1}$. We also estimate the stellar velocity dispersions from the NIR stellar CO (2-0) absorption feature for a subset of galaxies using SINFONI data and find them to be in the range $101 \leq \sigma_{\star \mathrm{CO}(2-0)} \leq 231 \mathrm{~km} \mathrm{~s}^{-1}$. For the galaxies for which we have both observations, the two stellar velocity dispersion measurements are in good agreement. On average, the stellar velocity dispersion derived from the NIR CO feature is higher by $\sim 3.69 \pm 0.93 \mathrm{~km} \mathrm{~s}^{-1}$ than the value derived from the CaT.

- We apply Monte Carlo-like simulations to test the robustness of stellar velocity dispersion estimations for bright AGNs in which we test the effects of $\mathrm{S} / \mathrm{N}$ and of the AGN continuum and emission lines. We conclude that stellar velocity dispersions can be obtained accurately for AGNs if the data have a $S / N>15$.

- We derive the SMBH masses of the LLAMA sample of Seyfert 1 AGNs from broad-line-based black hole mass estimates, which result in $6.34 \leq \log M_{\mathrm{BH}} \leq 7.75 M_{\odot}$ using the $\mathrm{H} \alpha$ line width and flux as a tracer of black hole mass. We additionally estimate $\mathrm{H} \beta$ emission line black hole masses for our sample of AGNs. When the $\mathrm{H} \beta$ was not available, we use the $\mathrm{Pa} \beta$ emission line instead (see Table 3 ).

- We find the Eddington ratio and accretion rates of the LLAMA sample to be within $0.004 \leq \lambda \leq 0.49$ and $0.04<\dot{M}<0.92 M_{\odot} \mathrm{yr}^{-1}$, respectively. The median for Type 1 and Type 2 is $\sim 0.08$ less than expected of Seyfert galaxies $(10 \%)$, but perhaps consistent with the selection method (hard X-ray).

- We find the best-fitting parameters for the LLAMA $M_{\mathrm{BH}}-\sigma_{\star}$ relation are $\alpha=8.14 \pm 0.20, \beta=3.38 \pm 0.65, \epsilon=0.32 \pm 0.06$. Within our uncertainties, the LLAMA AGN sample is consistent with the $M_{\mathrm{BH}}-\sigma \star$ relations reported by Woo et al. $(2013,2015)$ in terms of slope. The average intrinsic scatter of LLAMA sample around the Kormendy \& Ho (2013) $M_{\mathrm{BH}-\sigma \star}$ relation is found to be 0.30 dex. This intrinsic scatter is consistent with the intrinsic scatter of the Kormendy \& Ho (2013) $M_{\mathrm{BH}}-\sigma_{\star}$ relation. Correspondingly, we report that the pseudo-bulge-dominated LLAMA AGNs are now on the $M_{\mathrm{BH}}-\sigma_{\star}$ relation reported by Kormendy \& Ho (2013) (see the right panel of Fig. 5).

- We infer black hole masses for the other LLAMA Seyfert 2 AGNs as well as the inactive galaxies in the sample using the $M_{\mathrm{BH}}-\sigma_{\star}$ relation of the LLAMA AGNs with single-epoch $\mathrm{RM}$ or maser black hole masses.

- We argue that to reduce the offset from the ellipticaldominated $M_{\mathrm{BH}}-\sigma_{\star}$ relation, a correction to $M_{\mathrm{BH}}$ for the dust extinction (derived via the $\mathrm{H} \alpha$ or continuum flux) and a correction of $\sigma_{\star}$ for a rotational component of the disk/bulge must be applied to spiral-dominated local Seyfert AGNs.

Our main finding implies that the $M_{\mathrm{BH}}-\sigma_{\star}$ relation could be same for both elliptical and pseudo-bulge hosting galaxies. Correspondingly, we encourage further investigations with larger samples.

Acknowledgements. We would like to thank the anonymous referee for the comments and suggestions. TC would like to thank Hojin Cho, Stefano Marchesi, Federica Ricci, Julián Mejía-Restrepo, Murillo Marinello Assis de Oliveira, Swayamtrupta Panda, Nathen Nguyen, Kimberly Emig, Kirsty Butler, Fraser Evans, Dirk van Dam and Walter Jaffe for very useful discussions. T. C. is partly supported by a DFG grant within the SPP 1573 "Physics of the interstellar medium". R. A. R. thanks partial support from Conselho Nacional de Desenvolvimento Científico e Tecnológico and Fundação de Amparo à pesquisa do Estado do RS. S. V. acknowledges support from a Raymond and Beverley Sackler Distinguished Visitor Fellowship and thanks the host institute, the Institute of Astronomy, where this work was concluded. S. V. also acknowledges support by the Science and Technology Facilities Council (STFC) and by the Kavli Institute for Cosmology, Cambridge. VNB gratefully acknowledge assistance from a National Science Foundation (NSF) Research at Undergraduate Institutions (RUI) grant AST-1909297. Note that findings and conclusions do not necessarily represent views of the NSF.

\section{References}

Abuter, R., Schreiber, J., \& Eisenhauer, F. 2006, New A Rev., 50, 398 Akritas, M. G., \& Bershady, M. A. 1996, ApJ, 470, 706

Astropy Collaboration (Robitaille, T. P., et al.) 2013, A\&A, 558, A33

Astropy Collaboration (Price-Whelan, A. M., et al.) 2018, AJ, 156, 123

Baggett, W. E., Baggett, S. M., \& Anderson, K. S. J. 1998, AJ, 116, 1626

Rothberg, B., \& Fischer, J. 2010, ApJ, 712, 318

Baumgartner, W. H., Tueller, J., Markwardt, C., \& Skinner, G. 2010, BAAS, 42, 675

Barth, A. J., Greene, J. E., \& Ho, L. C. 2005, ApJ, 619, 151

Beifiori, A., Courteau, S., Corsini, E. M., \& Zhu, Y. 2012, MNRAS, 419, 2497

Batiste, M., Bentz, M. C., Raimundo, S. I., Vestergaard, M., \& Onken, C. A. 2017a, ApJ, 838, 10 
Batiste, M., Bentz, M. C., Manne-Nicholas, E. R., Onken, C. A., \& Bershady, M. A. 2017b, ApJ, 835, 271

Bentz, M. C., Denney, K. D., Cackett, E. M., et al. 2006, ApJ, 651, 775

Bentz, M. C., Walsh, J. L., Barth, A. J., et al. 2009a, ApJ, 705, 199

Bentz, M. C., Peterson, B. M., Netzer, H., Pogge, R. W., \& Vestergaard, M 2009b, ApJ, 697, 160

Bentz, M. C., Denney, K. D., Grier, C. J., et al. 2013, ApJ, 767, 149

Bentz, M. C., Cackett, E. M., Crenshaw, D. M., et al. 2016, ApJ, 830, 136

Bian, W.-H., \& Zhao, Y.-H. 2003, PASJ, 55, 599

Blandford, R., \& McKee, C. F. 1982, ApJ, 255, 419

Braatz, J., Condon, J., Constantin, A., et al. 2015, IAU Gen. Assem., 22, 2255730

Burtscher, L., Davies, R. I., Graciá-Carpio, J., et al. 2016, A\&A, 586, A28

Capetti, A. 2007, Balmaverde, B., 469, 88

Cappellari, M. 2017, MNRAS, 466, 798

Cappellari, M., \& Emsellem, E. 2004, PASP, 116, 138

Cappellari, M., Neumayer, N., Reunanen, J., et al. 2009, MNRAS, 394, 660

Chen, Y.-P., Trager, S. C., Peletier, R. F., et al. 2014, A\&A, 565, A117

Collin, S., \& Huré, J.-M. 2001, A\&A, 372, 50

Collin, S., Kawaguchi, T., Peterson, B. M., \& Vestergaard, M. 2006, A\&A, 456, 75

Combes, F., Garcá-Burillo, S., Audibert, A., et al. 2019, A\&A, 623, A79

Davidson, K. 1972, ApJ, 171, 213

Davies, R. I., Burtscher, L., Rosario, D., et al. 2015, ApJ, 806, 127

Davies, R. I., Hicks, E. K. S., Erwin, P., et al. 2017, MNRAS, 466, 4917

de Lapparent, V., Baillard, A., \& Bertin, E. 2011, A\&A, 532, A75

Denney, K. D., Bentz, M. C., Peterson, B. M., et al. 2006, ApJ, 653, 152

Denney, K. D., Peterson, B. M., Dietrich, M., Vestergaard, M., \& Bentz, M. C. 2009, ApJ, 692, 246

Denney, K. D., Peterson, B. M., Pogge, R. W., et al. 2010, ApJ, 721, 715

Eun, D.-I., Woo, J.-H., \& Bae, H.-J. 2017, ApJ, 842, 5

Falcón-Barroso, J., Lyubenova, M., van de Ven, G., et al. 2017, A\&A, 597, A48

Ferrarese, L., \& Ford, H. 2005, Space Sci. Rev., 116, 523

Ferrarese, L., \& Merritt, D. 2000, ApJ, 539, 9

Fisher, D. B., \& Drory, N. 2010, ApJ, 716, 2

Fisher, D. B., \& Drory, N. 2016, in Galactic Bulges, eds. E. Laurikainen, R. Peletier, \& D. Gadotti (Berlin: Springer), 41

Freudling, W., Romaniello, M., Bramich, D. M., et al. 2013, A\&A, 559, A96

Gadotti, D. A. 2008, MNRAS, 384, 420

Gebhardt, K., Bender, R., Bower, G., \& Stern, D. 2000, ApJ, 539, 13

Graham, A. W., Onken, C. A., Athanassoula, E., \& Combes, F. 2011, MNRAS, 412,2211

GRAVITY Collaboration (Sturm, E., et al.) 2018, Nature, 563, 657

Greene, J. E., \& Ho, L. C. 2005, ApJ, 630, 122

Greene, J. E., \& Ho, L. C. 2006a, ApJ, 641, 21

Greene, J. E., \& Ho, L. C. 2006b, ApJ, 641, 117

Greene, J. E., Peng, C. Y., \& Kim, M. 2010, ApJ, 721, 26

Greene, J. E., Seth, A., Kim, M., et al. 2016, ApJ, 826, 32

Grier, C. J., Peterson, B. M., Pogge, R. W., et al. 2012, ApJ, 755, 60

Grier, C. J., Peterson, B. M., Horne, K., et al. 2013a, ApJ, 764, 47

Grier, C. J., Martini, P., Watson, L. C., et al. 2013b, ApJ, 773, 90

Grier, C. J., Pancoast, A., Barth, A. J., et al. 2017, ApJ, 849, 146

González-Martín, O., Masegosa, J., Marquez, I., et al. 2015, A\&A, 578, A74

Gao, H., Ho, L. C., Barth, A. J., \& Li, Z.-Y. 2019, ApJS, 244, 34

Gu, Q., Melnick, J., Fernandes, R. C., et al. 2006, MNRAS, 366, 480

Gültekin, K., Richstone, D. O., Gebhardt, K., et al. 2009, ApJ, 698, 198

Harris, C. E., Bennert, V. N., Auger, M. W., et al. 2012, ApJS, 201, 29

Hu, C., Wang, J.-M., Ho, L. C., et al. 2016, ApJ, 832, 197

Kang, W.-R., Woo, J.-H., Schulze, A., et al. 2013, ApJ, 767, 26

Kaspi, S., Smith, P. S., Netzer, H., et al. 2000, ApJ, 533, 631

King, A. 2003, ApJ, 596, 27

Knapen, J. H., de Jong, R. S., Stedman, S., \& Bramich, D. M. 2003, MNRAS, 344,527

Koss, M., Trakhtenbrot, B., Ricci, C., et al. 2017, ApJ, 850, 74

Kormendy, J., \& Ho, C. L. 2013, ARA\&A, 51, 511

Kormendy, J., Bender, R., \& Cornell, M. N. 2011, Nature, 469, 374

Kormendy, J., Drory, N., Bender, R., \& Cornell, M. E. 2015, ApJ, 723, 1

Kozłowski, S. 2017, ApJS, 228, 9

La Franca, F., Onori, F., Ricci, F., et al. 2015, MNRAS, 449, 1526

Landt, H., Ward, M. J., Peterson, B. M., et al. 2013, MNRAS, 432, 113

Laurikainen, E., Salo, H., Buta, R., Knapen, J. H., \& Comerón, S. 2010 MNRAS, 405, 1089

Lynden-Bell, D. 1969, Nature, 223, 690

Lin, M.-Y., Davies, R. I., Hicks, E. K. S., et al. 2018, MNRAS, 473, 4582

Marinucci, A., Bianchi, S., Nicastro, F., Matt, G., \& Goulding, A. D. 2012, ApJ, 748,130

McConnell, N. J., \& Ma, C.-P. 2013, ApJ, 764, 184

Maiolino, R., \& Rieke, G. H. 2013, ApJ, 454, 95
Merritt, D., \& Ferrarese, L. 2001, ApJ, 547, 140

Mejía-Restrepo, J. E., Trakhtenbrot, B., Lira, P., Netzer, H., \& Capellupo, D. M. 2016, MNRAS, 460, 187

Mejía-Restrepo, J. E., Lira, P., Netzer, H., Trakhtenbrot, B., \& Capellupo, D. M. 2018, Nat. Astron., 2, 63

Modigliani, A., Goldoni, P., Royer, F., et al. 2010, Proc. SPIE, 7737, 28

Nelson, C. H., Green, R. F., Bower, G., Gebhardt, K., \& Weistrop, D. 2004, ApJ, 615,652

Netzer, H. 1990, in Active Galactic Nuclei, eds. R. D. Blandford, H. Netzer,

L. Woltjer, T. J. L. Courvoisier, \& M. Mayor (Berlin: Springer-Verlag), 57

Netzer, H., Elitzur, M., \& Ferland, G. J. 1990, ApJ, 299, 752

Neumayer, N. 2010, PASA, 27, 449

Oke, J. B., Shields, G. A., \& Korycansky, D. G. 1984, ApJ, 277, 64

Onken, C. A., \& Peterson, B. M. 2002, ApJ, 572, 746

Onken, C. A., Ferrarese, L., Merritt, D., et al. 2004, ApJ, 615, 645

Osterbrock, D. E., \& Ferland, G. J. 2006, Astrophysics of Gaseous Nebulae and Active Galactic Nuclei (Sausalito, CA: University Science Books)

Osterbrock, D. E., \& Pogge, R. W. 1985, ApJ, 297, 166

Padovani, P., Alexander, D. M., Assef, R. J., et al. 2017, A\&ARv, 25, 2

Pancoast, A., Brewer, B. J., Treu, T., et al. 2014, MNRAS, 445, 3073

Park, D., Kelly, B. C., Woo, J.-H., \& Treu, T. 2012a, ApJS, 203, 6

Park, D., Woo, J.-H., Treu, T., et al. 2012b, ApJ, 747, 30

Peterson, B. M. 1993, PASP, 105, 247

Peterson, B. M. 2007, ASP Conf. Ser., 373, 3

Peterson, B. M. 2011, Proceedings of the Workshop "Narrow-Line Seyfert Galaxies and their Place in the Universe", PoS(NLS1)032

Peterson, B. M., \& Bontà, E. D. 2018, Proceedings of the Workshop "NarrowLine Seyfert Galaxies and their Place in the Universe", PoS(NLS1)032

Peterson, B. M., Ferrarese, L., Gilbert, K. M., et al. 2004, ApJ, 613, 682

Press, W. H., Teukolsky, S. A., Vetterling, W. T., \& Slannery, B. P. 1992, Numerical Recipes in C. The Art of Scientific Computing, Second Edition (Cambridge, New York, New Rochelle, Melbourne and Sydney: Cambridge University Press)

Rosario, D. J., Burtscher, L., Davies, R. I., et al. 2018, MNRAS, 473, 5658

Rosario, D. J., Togi, A., Burtscher, L., et al. 2019, ApJ, 875, 8

Ricci, C., Trakhtenbrot, B., Koss, M. J., et al. 2017a, ApJS, 233, 17

Ricci, F., La Franca, F., Onori, F., \& Bianchi, S. 2017b, A\&A, 598, A51

Riffel, R. A., Ho, L. C., Mason, R., et al. 2015, MNRAS, 446, 2823

Rybicki, G. B., \& Lightman, A. P. 1986, Radiative Processes in Astrophysics (New York: Wiley-VCH), 400

Saglia, R. P., Opitsch, M., Erwin, P., et al. 2016, ApJ, 818, 47

Salo, H., Laurikainen, E., Laine, J., et al. 2015, ApJS, 219, 4

Shimizu, T. T., Davies, R. I., Koss, M., et al. 2018, ApJ, 856, 154

Shimizu, T. T., Davies, R. I., Lutz, D., et al. 2019, MNRAS, 490, 5860

Silge, J. D., \& Gebhardt, K. 2003, AJ, 125, 2809

Silk, J., \& Rees, M. J. 1998, A\&A, 331, 1

Schnorr-Müller, A., Davies, R. I., Korista, K. T., et al. 2016, MNRAS, 462, 3570

Shakura, N. I., \& Sunyaev, R. A. 1973, A\&A., 24, 337

Shankar, F., Bernardi, M., \& Sheth, R. K. 2016, MNRAS, 460, 3119

Shankar, F., Bernardi, M., Richardson, K., et al. 2019, MNRAS, 485, 1278

Shen, Y., \& Liu, X. 2012, ApJ, 753, 125

Shen, J., Vanden Berk, D. E., Schneider, D. P., \& Hall, P. B. 2008, AJ, 135, 928

Storchi-Bergmann, T., \& Schnorr-Müller, A. 2019, Nat. Astron., 3, 48

Storchi-Bergmann, T., Schimoia, J. S., Peterson, B. M., et al. 2017, ApJ, 835 , 236

Subramanian, S., Ramya, S., Das, M., et al. 2016, MNRAS, 455, 3148

Skrutskie, M. F., Cutri, R. M., Stiening, R., et al. 2006, AJ, 131, 1163

Terlevich, E., Diaz, A. I., \& Terlevich, R. 1990, MNRAS, 242, 271

Tremaine, S., Gebhardt, K., Bender, R., et al. 2002, ApJ, 574, 740

Tully, R. B., \& Fisher, J. R. 1977, A\&A, 54, 661

Vanden Berk, D. E., Richards, G. T., Bauer, A., et al. 2001, AJ, 122, 549

van den Bosch, R. C. E., Gebhardt, K., Gültekin, K., Yıldırım, A., \&

Walsh, J. L. 2015, ApJS, 218, 10

van den Bosch, R. C. E. 2016, ApJ, 831, 134

van der Marel, R. P., \& Franx, M. 1993, ApJ, 407, 525

Véron, M.-P., \& Véron, P. 2010, A\&A, 518, A10

Wada, K. 2012, ApJ, 758, 66

Wandel, A., Peterson, B. M., \& Malkan, M. A. 1999, ApJ, 526, 579

Vestergaard, M., \& Peterson, B. M. 2006, ApJ, 641, 689

Wild, V., Groves, B., Heckman, T., et al. 2011, MNRAS, 410, 1593

Winge, C., Riffel, R. A., \& Storchi-Bergmann, T. 2009, ApJS, 185, 186

Winter, L., Veilleux, S., McKernan, B., \& Kallman, T. 2012, ApJ, 745, 107

Woo, J.-H., Treu, T., \& Barth, A. J. 2010, ApJ, 716, 269

Woo, J.-H., Schulze, A., Park, D., et al. 2013, ApJ, 772, 49

Woo, J.-H., Yoon, Y., Park, S., Park, D., \& Kim, S. C. 2015, ApJ, 801, 38

Yoshino, A., \& Yamauchi, C. 2015, MNRAS, 446, 3749

Yu, Q., \& Lu, Y. 2004, ApJ, 610, 93 


\section{Appendix A: Broad-Line fittings}
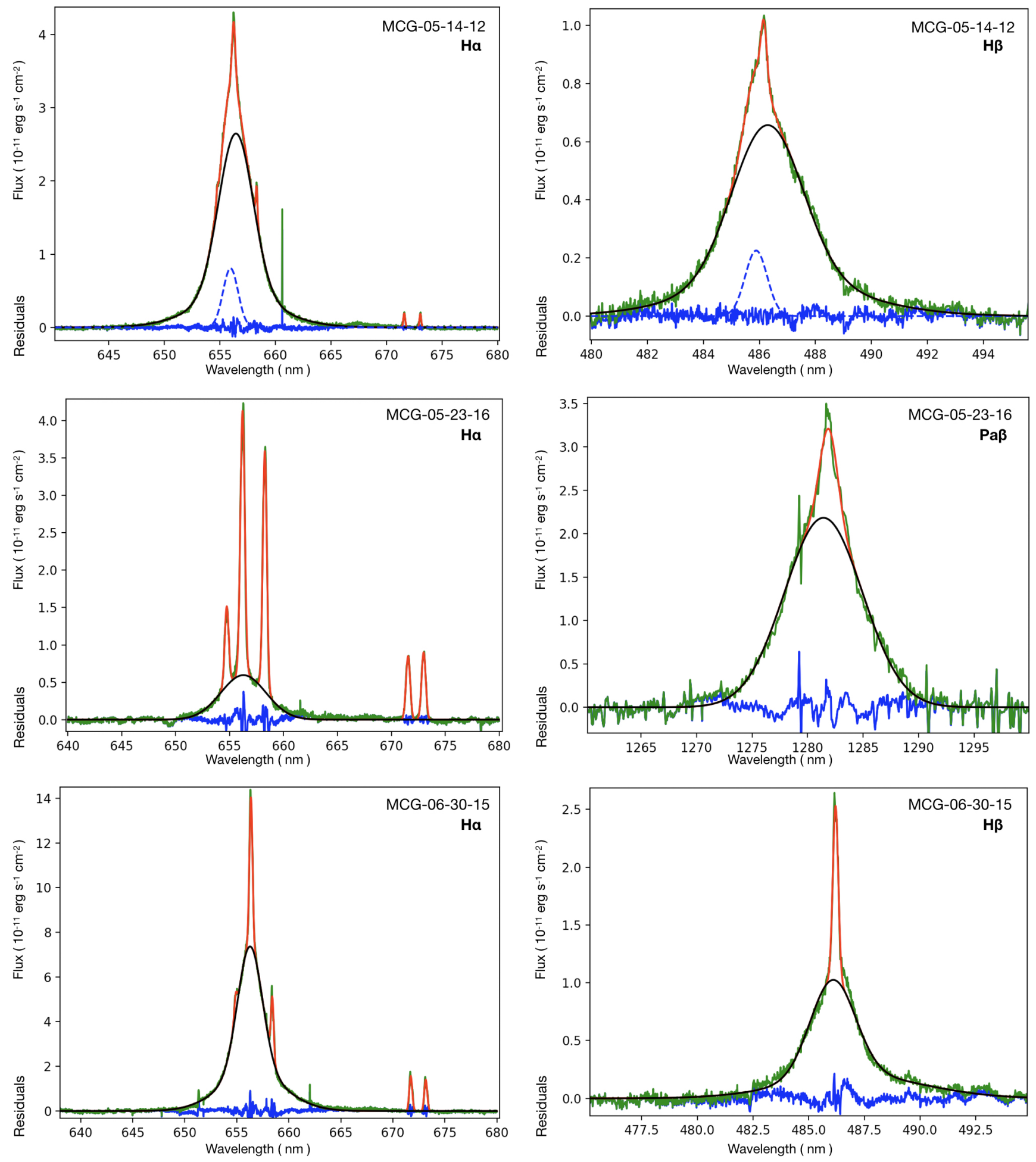

Fig. A.1. Broad-line region emission fittings of our sample. The black solid line represents the broad-line emission line width, whereas the red solid line represents the best fit. Residuals are shown in blue for visual aids. The unidentified blue-shifted broad emission lines of NGC 1365, NGC 2992 and MCG-05-14-12 are presented as blue dashed line. 

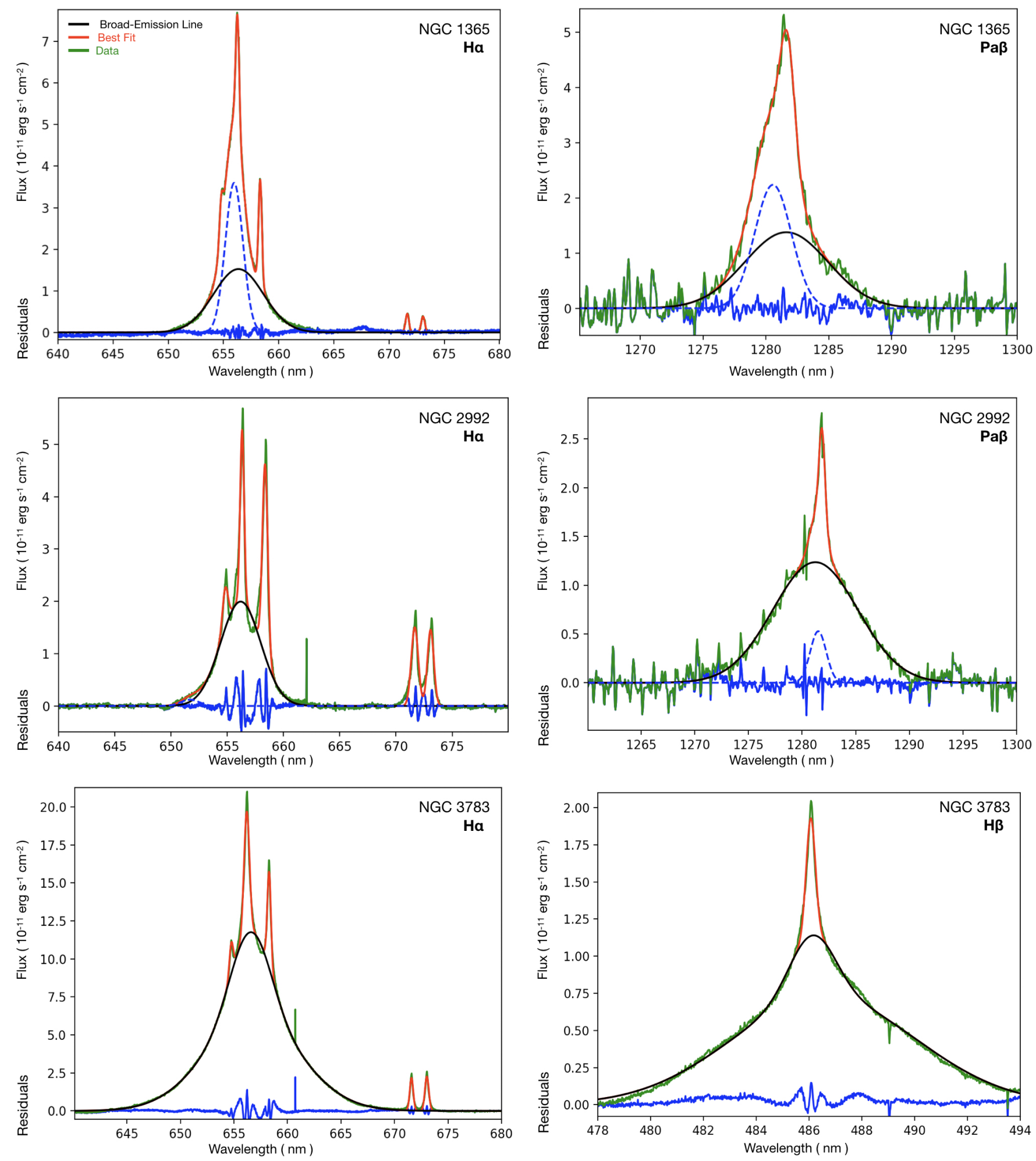

Fig. A.1. continued. 
T. Caglar et al.: LLAMA: The $M_{\mathrm{BH}}-\sigma_{\star}$ relation of the most luminous local AGNs
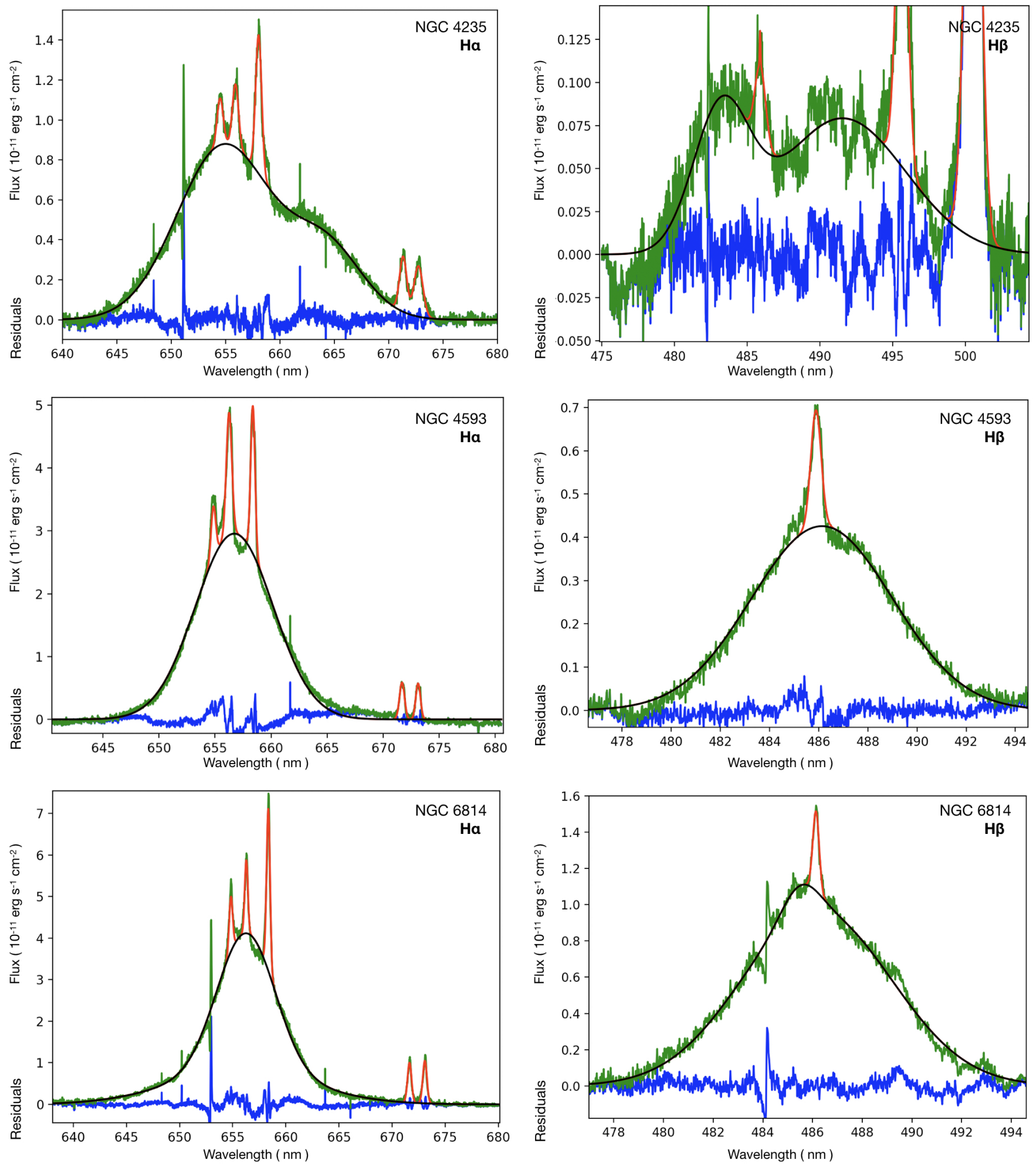

Fig. A.1. continued. 
A\&A 634, A114 (2020)
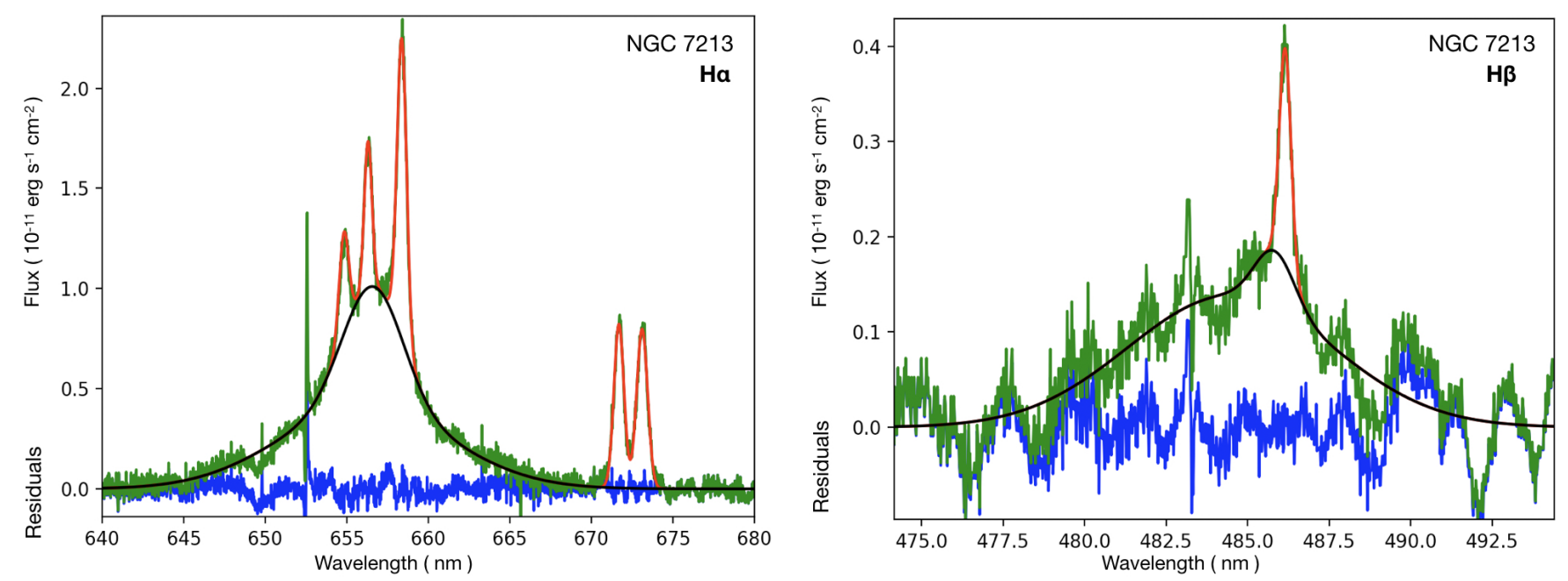

Fig. A.1. continued. 
T. Caglar et al.: LLAMA: The $M_{\mathrm{BH}}-\sigma_{\star}$ relation of the most luminous local AGNs

\section{Appendix B: The pPXF fittings}

We present the pPXF stellar velocity dispersion fitting results from CaT absorption lines (from Fig. B.1), whereas CO (2-0)

fitting results are presented from Fig. B.2.
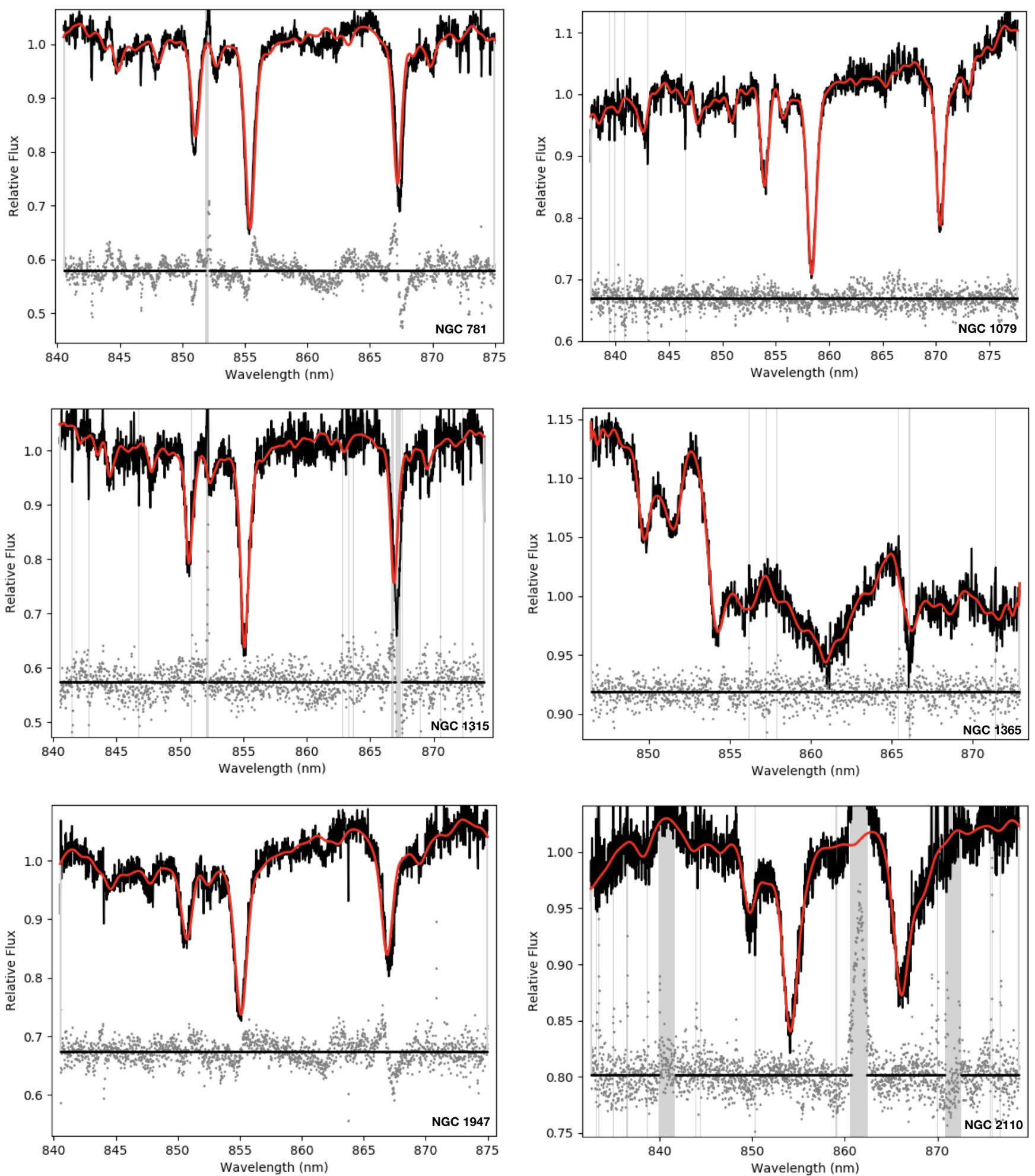

Fig. B.1. Fitting plots via pPXF for CaT. The red solid line represents the best fit, whereas the residuals are shown as gray. The vertical gray lines represent masked features. 

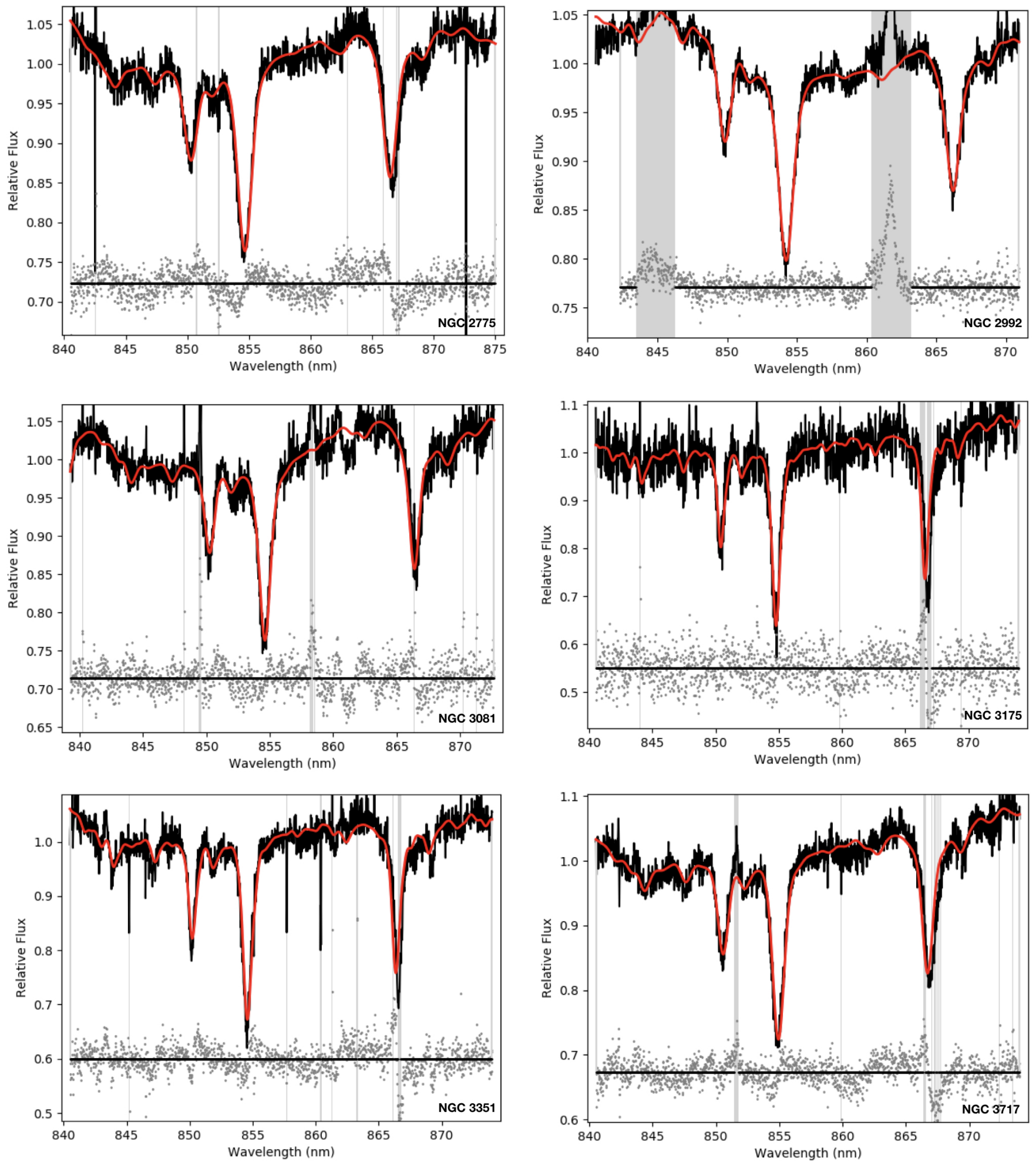

Fig. B.1. continued. 
T. Caglar et al.: LLAMA: The $M_{\mathrm{BH}}-\sigma_{\star}$ relation of the most luminous local AGNs
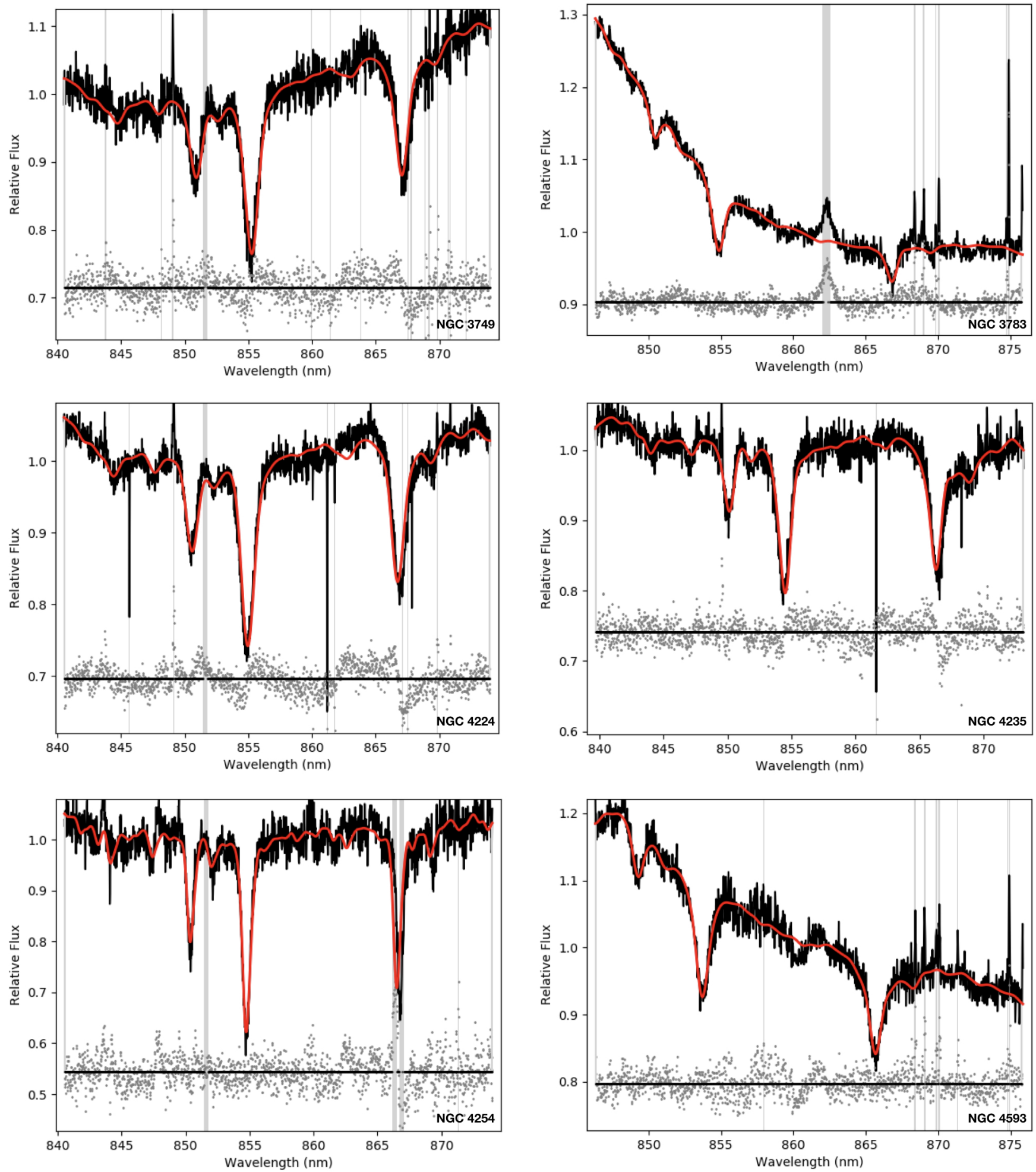

Fig. B.1. continued. 

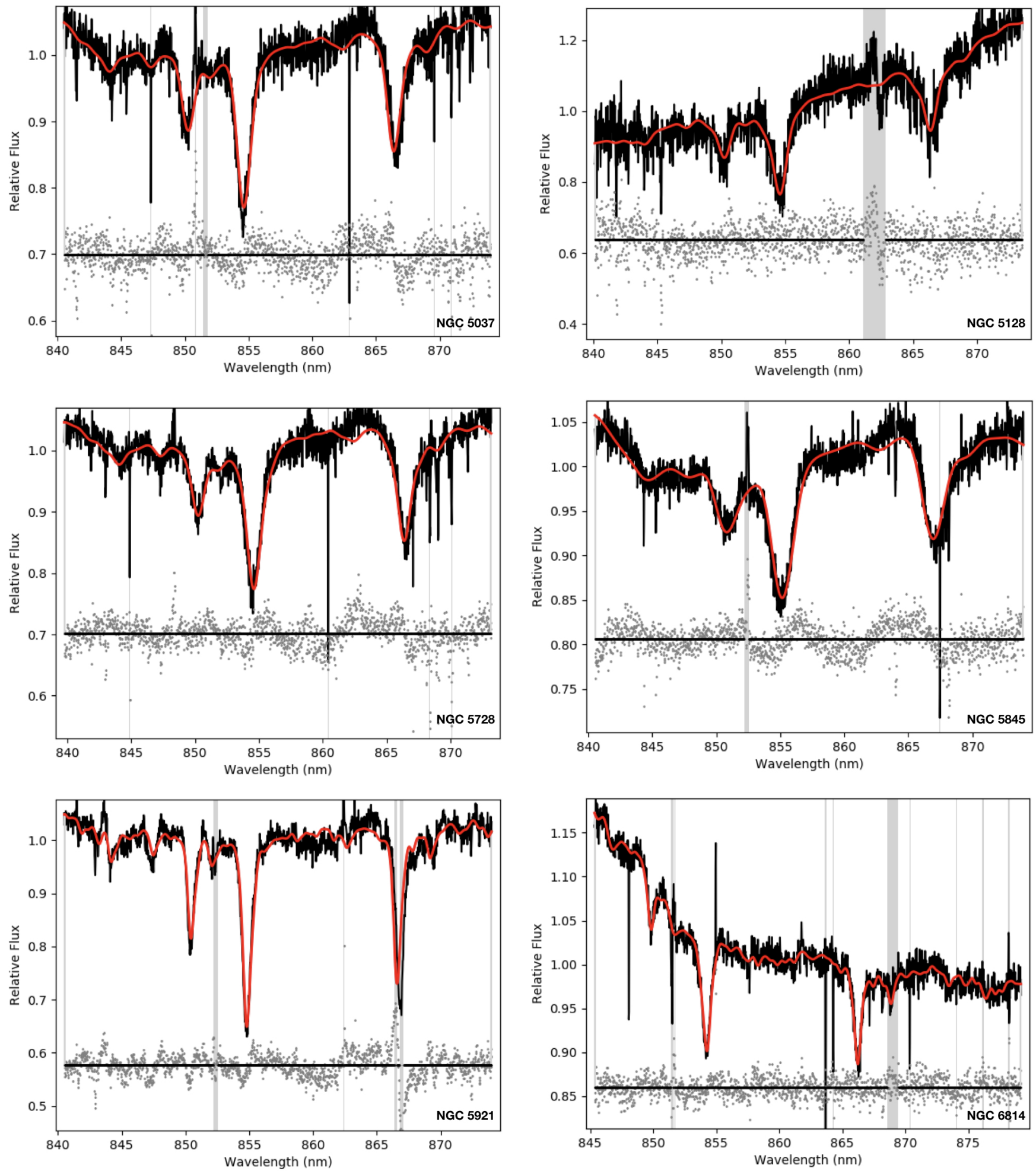

Fig. B.1. continued. 
T. Caglar et al.: LLAMA: The $M_{\mathrm{BH}}-\sigma_{\star}$ relation of the most luminous local AGNs
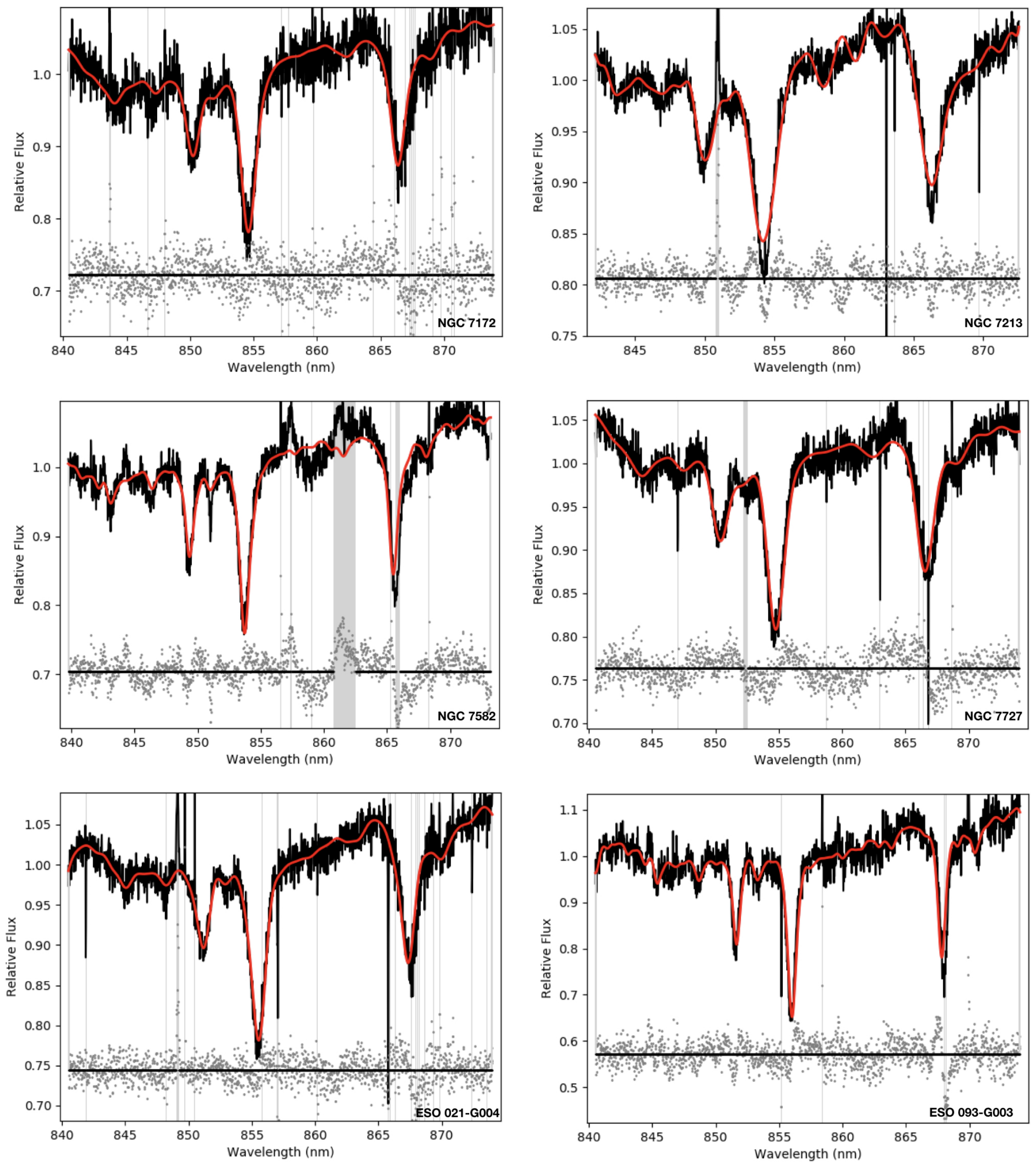

Fig. B.1. continued. 

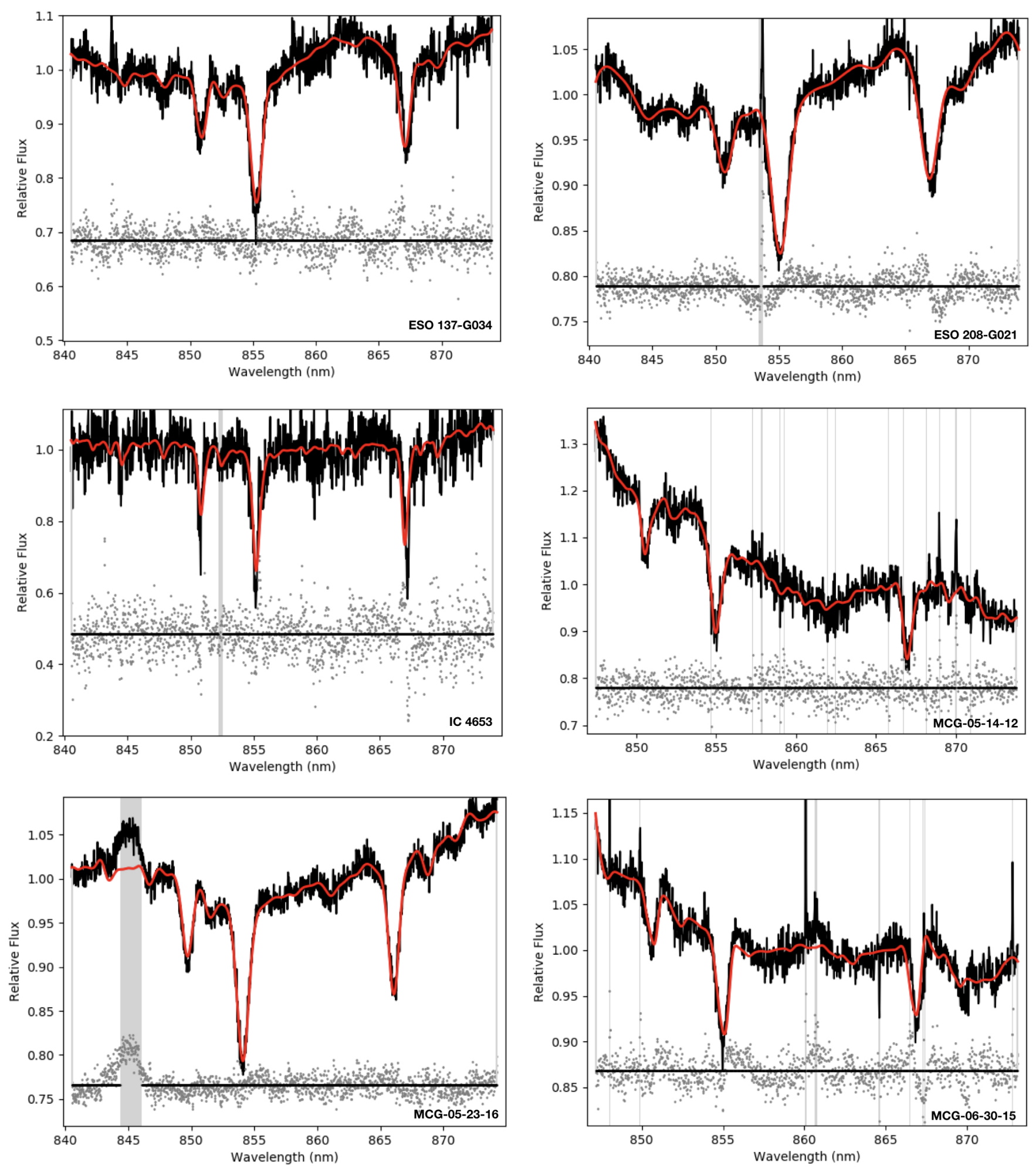

Fig. B.1. continued. 
T. Caglar et al.: LLAMA: The $M_{\mathrm{BH}}-\sigma_{\star}$ relation of the most luminous local AGNs
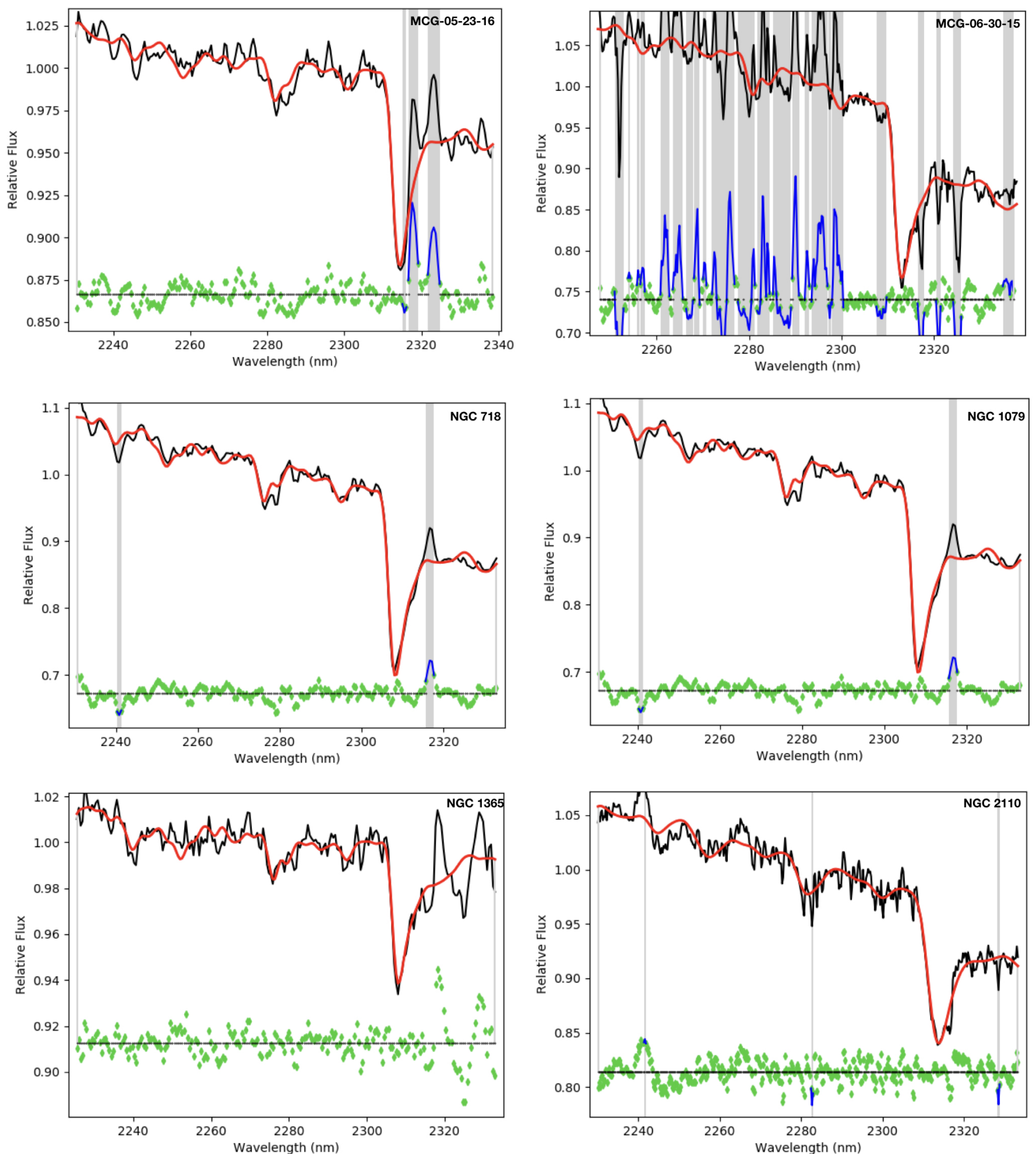

Fig. B.2. Fitting plots via pPXF for CO (2-0). The red solid line represents the best fit, whereas the residuals are shown as green. The vertical gray lines represent masked features. 

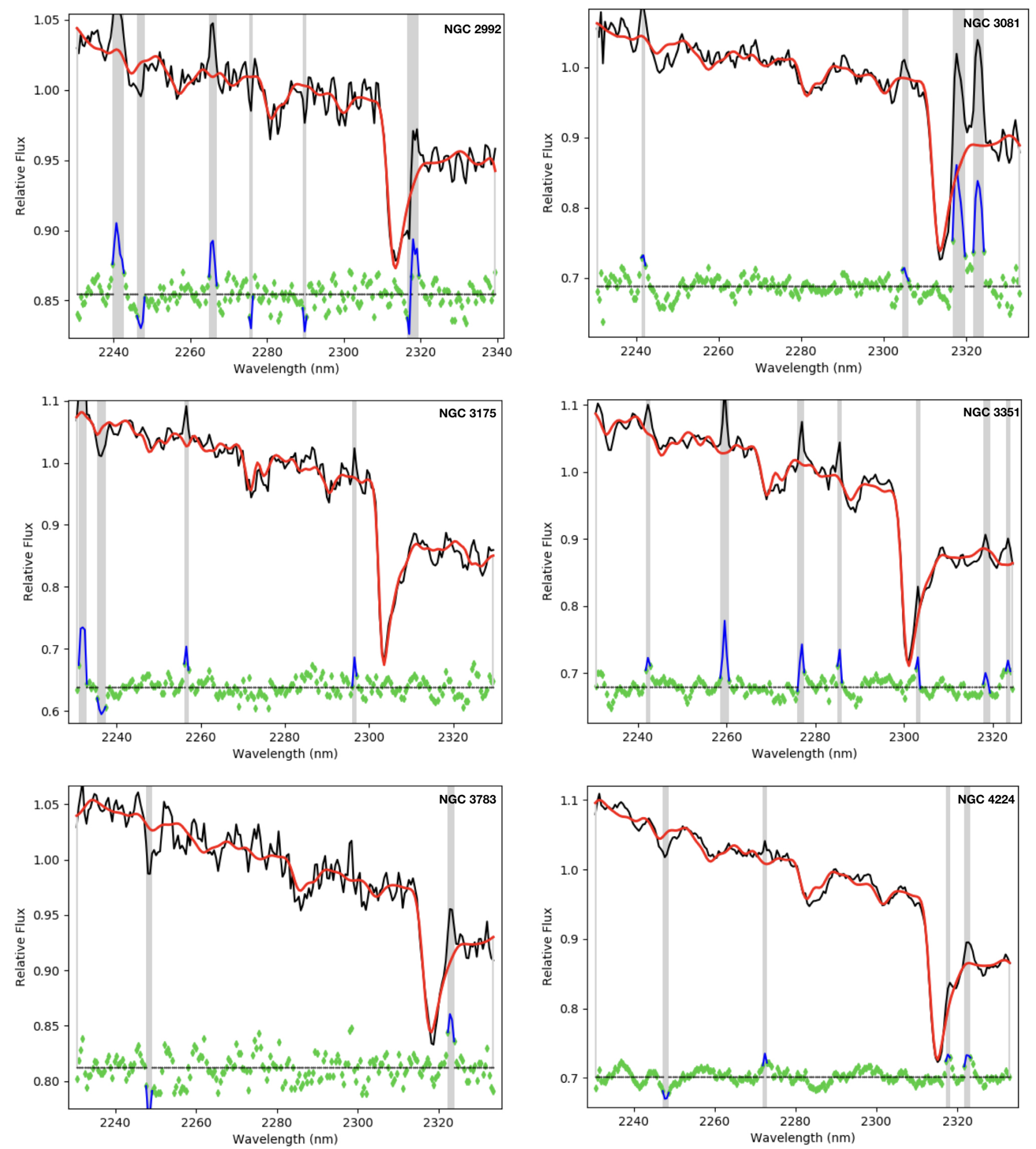

Fig. B.2. continued. 
T. Caglar et al.: LLAMA: The $M_{\mathrm{BH}}-\sigma_{\star}$ relation of the most luminous local AGNs
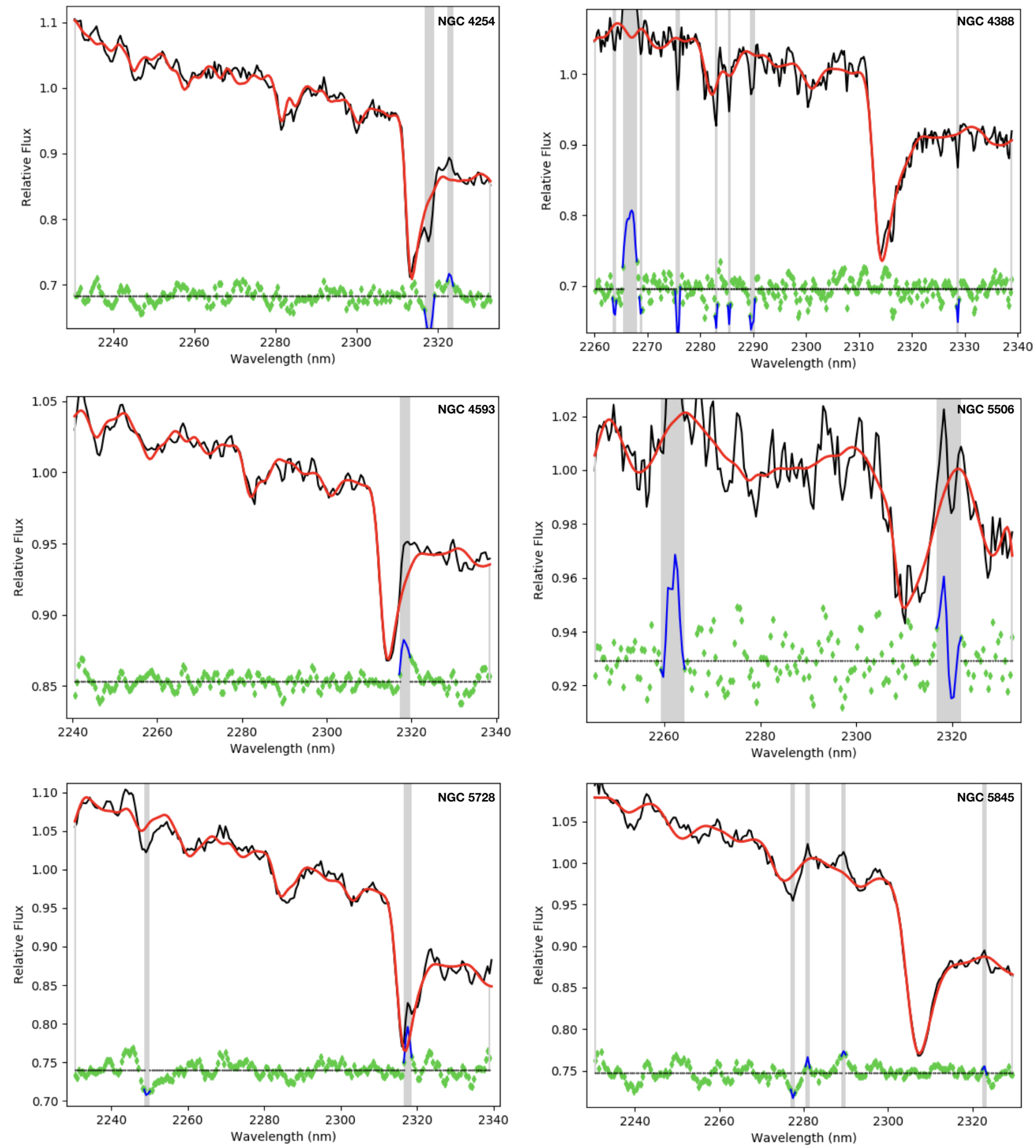

Fig. B.2. continued. 

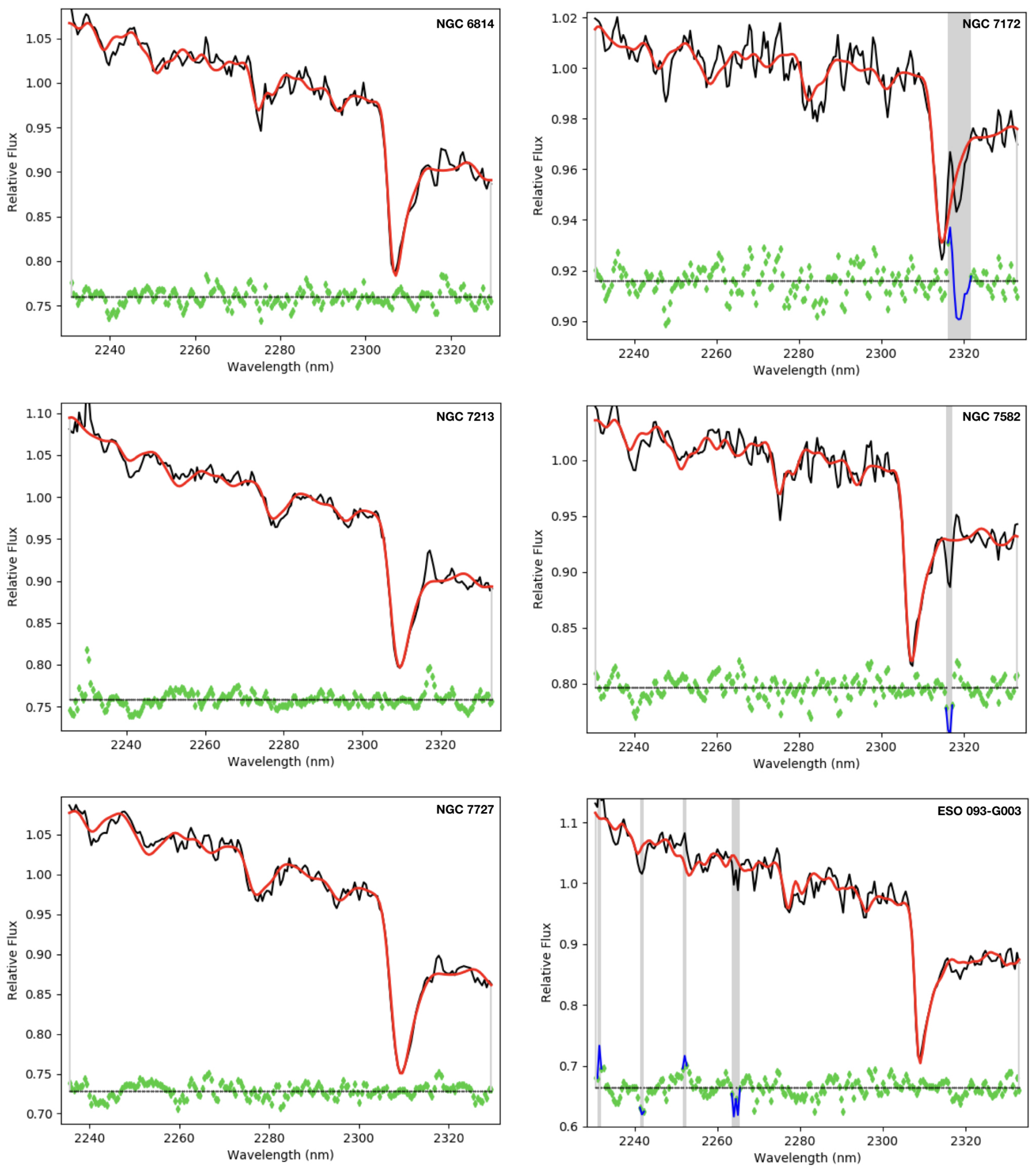

Fig. B.2. continued. 
T. Caglar et al.: LLAMA: The $M_{\mathrm{BH}}-\sigma_{\star}$ relation of the most luminous local AGNs
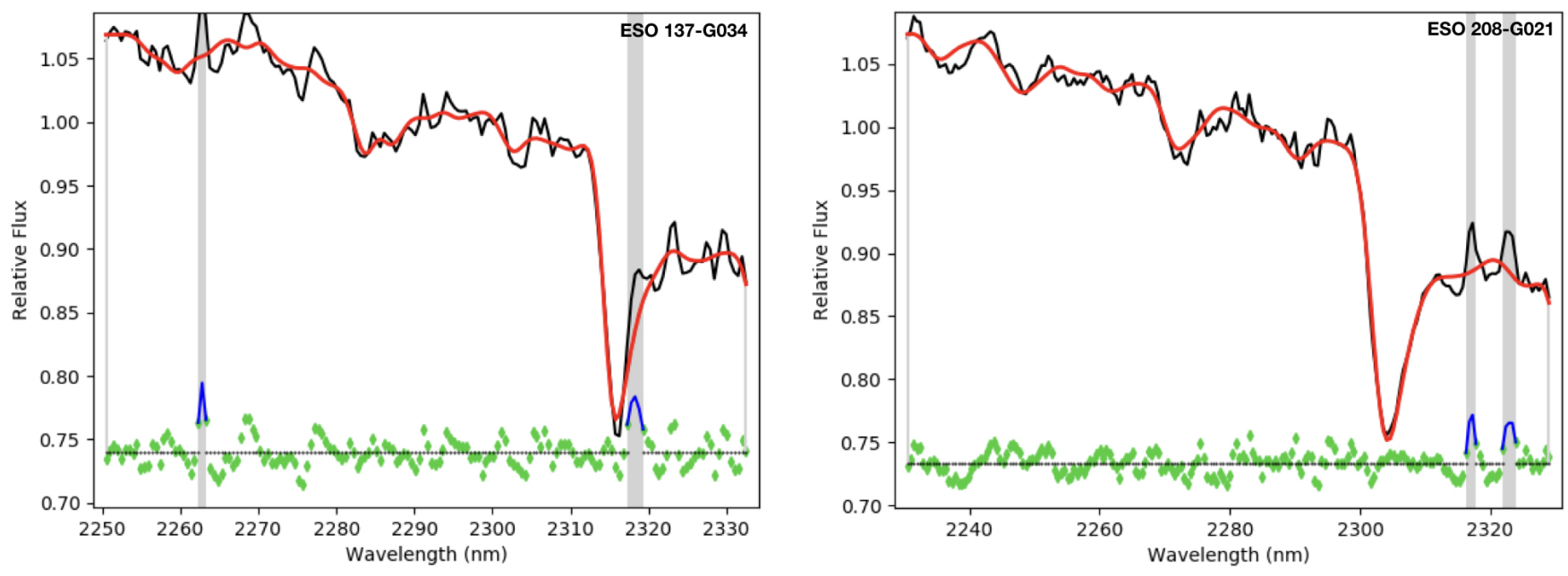

Fig. B.2. continued. 